\title{
Kapitel 4. \\ Literarische Transformationen - Ampelos im Spiegel mythologischer Dichtung
}

\author{
Es wird von Beroe der schönen viel gesagt, \\ Die auch Neptuno lieb, wie sehr sie dir behagt. \\ Wie Ampeli gelb' haar von Zephyrus getrieben \\ Dein unerlescht gemüth beweget hat zum lieben. \\ Wie daß dir durch den Stier und seinen grimmen todt, \\ Dadurch er weggerafft, erregt ward weh und noth. \\ Du hast das gülden' Haar von Ampelus genommen, \\ Verendert in die pflantz' auß der der wein herkommen: \\ Durch welches zue der Stund das Elend Weh und schmertz \\ Und leidige verdruß verließ dein traurig Hertz.
}

Martin Opitz ${ }^{1}$

\subsection{Ampelos zwischen Tradition und Innovation}

Für die Ampelos-Erzählung komponiert Nonnos eine Figur, die - außer in wenigen literarischen und bildlichen Zeugnissen - in der mythologischen Überlieferung nicht greifbar ist. Dafür, dass es sich dennoch nicht um eine völlig freie Erfindung handelt, spricht die Zusammenstellung und Anwendung wohlbekannter Motive und Motivelemente: Ampelos ist ein Satyr im Gefolge des Dionysos, der in seiner strahlenden Schönheit unerreichte Liebling des Gottes, der so weit geht, dass er sich göttliche Unsterblichkeit anmaßt, indem er sich in bakchischer Kleidung zeigt, und der als Konsequenz dieser Hybris mit dem Tode bestraft wird. Sein gewaltsamer Tod durch den Stier, das dionysische Lebewesen par excellence, und seine Wiederauferstehung in neuer Daseinsform macht Ampelos zu einem Grenzgänger zwischen irdischer und göttlicher Sphäre: Durch Metamorphose wird er ein Teil des Gottes selbst, in dem er

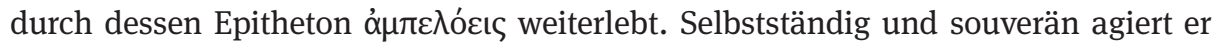
lediglich ein einziges Mal, wo er an den Göttern Dionysos und Selene Hybris begeht und so selbst zum Urheber seines tragischen Schicksals wird.

Dennoch sind bei aller Originalität und Gestaltungsfreiheit des Dichters Ampelos' hybrides Verhalten und zwangsläufiges Scheitern symptomatisch, ja topisch: Der junge Satyr erinnert an den Typus des männlichen, jugendlichen Göttergeliebten, der durch seine herausragende Schönheit die Aufmerksamkeit eines Gottes erregt, zu dessen Geliebten avanciert, aufgrund eines Fehltritts sein Leben verliert und letztlich zu den Unsterblichen aufsteigt. Beispiele für derartige Schicksale finden sich bereits

1 Opitz (1622). 
bei Homer, in der frühgriechischen Lyrik und in der hellenistischen Dichtung. Der Dichter aus Panopolis nimmt häufig wörtliche Anleihen bei Autoren aus allen Phasen der griechischen Dichtung, oftmals überträgt er auch Szenen, Themen und Motive und passt diese in den Kontext seines Epos ein. Seine in der literarischen Tradition blass und farblos erscheinende Ampelos-Figur schöpft der Dichter nur auf den ersten Blick aus dem literarischen Nichts, vielmehr bietet er eine große Zahl an mythischen Figuren zur Gegenüberstellung mit seinem Satyrn auf, wobei er die Parallelfiguren mit Bedacht auswählt und nichts dem Zufall überlässt: Für jede der aus der antiken literarischen Tradition geläufigen Gestalten lässt sich zumindest eine Gemeinsamkeit festmachen, durch die eine direkte Verbindungslinie zu Ampelos' Schicksal gezogen werden kann. ${ }^{2}$ Dieselben Thematiken und Motive erscheinen in immer wieder unterschiedlichen Kontexten, aber mit voneinander abweichenden Schwerpunktsetzungen. Die Entsprechungen der Vergleichspunkte erlauben eine Systematisierung der Figuren in fünf Themengruppen: (1.) Figuren, die sich durch Göttlichkeit und Unsterblichkeit auszeichnen, (2.) Götterlieblinge von außergewöhnlicher Schönheit, (3.) Figuren, die mit Hybris und Tod, (4.) Metamorphose und Wiederauferstehung sowie (5.) mit einer Pflanze oder einem daraus gewonnenen Produkt in Zusammenhang stehen.

Am Bauplan des Charakterprofils des Ampelos, welches sich in den Dionysiaka beinahe zur Gänze aus der Gegenüberstellung mit anderen, wohlbekannten mythischen Gestalten speist, werden die literarischen Techniken und poetischen Prinzipien des spätantiken Dichters greifbar. Im Folgenden gilt es zunächst zu demonstrieren, wie Ampelos mit diesen Figuren abgeglichen wird, um so dessen Charakterzeichnung herauszuarbeiten und den in der mythisch-literarischen Tradition weitgehend unbekannten Satyrn genauer zu umreißen und fassbar zu machen. Darüber hinaus soll Nonnos' poetische Verfahrensweise im Hinblick auf die Konzeption und Präsentation dieser Vergleichsfiguren beleuchtet werden: Welche Figuren dienen Ampelos zum Vergleich, und worin bestehen die Vergleichspunkte? An welchen Autoren orientiert sich Nonnos bei seinen Übernahmen, und in welcher Weise modifiziert und adaptiert er diese Vorbilder für seinen neuen Kontext? Wie fügen sich die einzelnen Vergleiche in die neue Erzählung um Ampelos ein? Lässt sich gar ein Netz aus lexikalischen und motivischen Bezügen aufspannen, für welches die Vergleichsfiguren als Knotenpunkte des narrativen Designs dienen?

2 Die hohe Dichte an Vergleichsfiguren, die Nonnos für Ampelos heranzieht, lässt das rhetorische Genus der Synkrisis durchscheinen. Der in der klassischen $\pi \alpha$ เ $\delta \varepsilon^{\prime} \alpha$ geschulte spätantike Dichter verfügte demnach über profunde Kenntnisse in der Rhetorik und benutzte rhetorische Gesetzmäßigkeiten und Stilprinzipien für das poetische Konzept seines Dionysos-Epos. Zum Einfluss der unterschiedlichen rhetorischen Genera auf die Dionysiaka vgl. Kap. 6, zur Synkrisis im Besonderen siehe Kap. 6.3. - Zu den Vergleichsfiguren für Ampelos siehe auch Carvounis (2016). 


\subsection{Ampelos und die Götter}

Ampelos wird zunächst vier Göttern gegenübergestellt und in der ersten Rede des Dionysos in Nonn. D. 10,196-216 mit drei dieser Götter verglichen: Eros (Nonn. D. 10,198-200), Hermes (203f.) und Apoll (205-207). ${ }^{3}$ Der augenscheinlichste Vergleichspunkt ist die Göttlichkeit der Figuren, die auf Ampelos übertragen und über die ihnen zugeschriebenen Attribute zum Ausdruck gebracht wird, wobei gleichsam ex

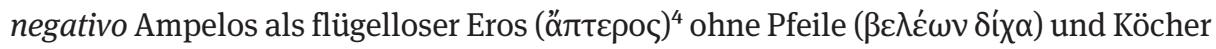

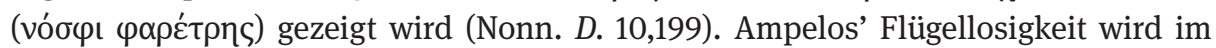
Folgenden erneut aufgegriffen, und zwar im Vergleich mit Hermes, der durch „leichte

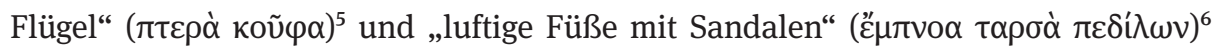
kenntlich gemacht wird (Nonn. D. 10,204). Das Thema Pfeil und Bogen wird auch im

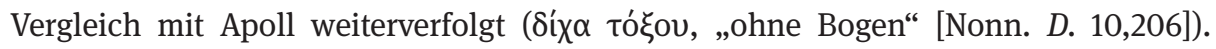
Wenngleich der Angesprochene über keines dieser Attribute verfügt, erhält er doch göttlichen Status zugesprochen, ja er übertrifft die genannten Götter, wenn er sogar ohne Erkennungszeichen in seiner Göttlichkeit sichtbar wird. Die Kombination von Attributlosigkeit und göttlicher Wirkkraft nimmt zudem die Fähigkeiten des Dionysos in den Indien-Kämpfen vorweg, der ohne besondere Kampfausrüstung dennoch seine verheerende göttliche Macht zum Einsatz bringt.

Durch den Vergleich mit den drei Göttern wird Ampelos mit außergewöhnlicher Schönheit und Jugend versehen. Jugendlichkeit ist für alle drei Götter signifikant, insbesondere für Apoll, der stets mit langem, offenem Haar gezeigt wird, so auch hier

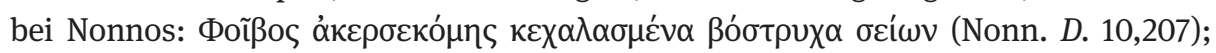
direkt mit Apoll wird Ampelos verglichen, wenn er in Nonn. D. 10,205 ebenfalls mit

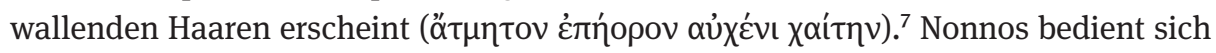
bei der Charakterisierung des Gottes Apoll traditioneller Diktion: Das Epitheton

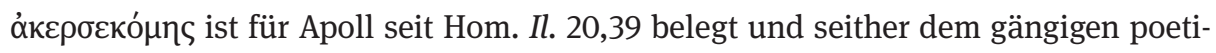

$3 \mathrm{Zu}$ Hermes in den Dionysiaka siehe Fayant (1998).

4 Das Adjektiv ö $\pi \tau \varepsilon \rho$, ,flügellos“, findet sich viermal in den Dionysiaka, vgl. Nonn. D. 4,87 und Nonn. D. 35,239 von Hermes und Nonn. D. 47,658 von Dionysos, sowie einmal in der Paraphrase, vgl. Хрıбто̀ है P. 20,119f.). Die Angleichung des Geliebten an einen flügellosen Eros ist ein Motiv der erotischen Literatur, vgl. AP 12,75 - 78; Ov. met. 4,321; Nonn. D. 4,238-241. Für Nonnos ist Eros für gewöhnlich mit Flügeln ausgestattet, vgl. Chrétien (1985) 145 Anm. ad 199; zu den Epitheta für Eros in den Dionysiaka siehe Martínez (2007) 78f. - Die in Nonn. D. 10,200 genannte Aphrodite als mutmaßliche Mutter des Ampelos hebt die Vergleichspunkte Schönheit und erotische Ausstrahlung zusätzlich heraus.

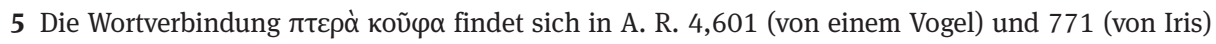
sowie mehrmals bei Nonnos, vgl. Nonn. D. 2,126; 4,2 u. ö.; Jo. Gaz. 1,352; vgl. auch in christlichem Zusammenhang: Synes. hymn. 1,700; Chrys. hom. 2,1,52 in Is. 6,1 (Dumortier).

6 Bei $\tau \alpha \rho \sigma \alpha ̀ ~ \pi \varepsilon \delta i ́ \lambda \omega v$ handelt es sich um eine nonnianische Formel - vgl. Nonn. D. 9,93; 11,490;

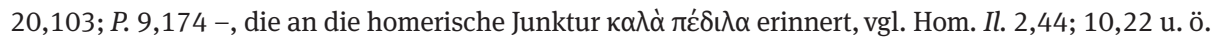

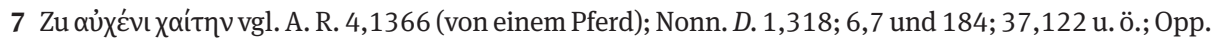
C. 3,255; Jo. Gaz. 2,19. 
schen Wortschatz zugehörig. ${ }^{8}$ Auch für die „traubenförmigen Locken“ greift Nonnos auf ein Vorbild zurück: Auf die Landung der Argonauten an der Tyneïschen Insel folgt

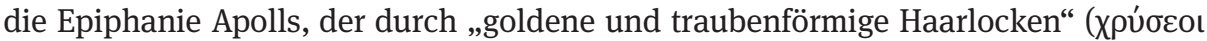

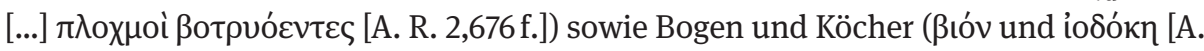
R. 2,678f.]) kenntlich gemacht wird. Vor dem Hintergrund der Stellen in der Ilias und in den Argonautika bietet Nonnos gleichsam die Epiphanie seines Satyrn Ampelos, der nun zum ersten Mal in den Dionysiaka in Erscheinung tritt und dessen vorherbestimmte Unsterblichkeit durch subtile intertextuelle Anspielungen von Beginn an betont wird.

Nonnos formt den Satyrn nach dem Ausschließungsprinzip, ihm gelingt durch die katalogartige Aufzählung, was Ampelos nicht ist, eine erste Annäherung an sein Wesen. Der Dichter schreibt dem Satyrn Schönheit, Jugend und Göttlichkeit zu, indem er sich vertrauter Elemente und Gemeinplätze der griechischen Dichtung bedient. Mit den bewusst gewählten wörtlichen Zitaten und der Anlehnung an die epische Formensprache knüpft der spätantike Dichter an seine Vorgänger an, zeigt Vertrautes jedoch in neuem Licht, indem er die Inhalte der zugrunde liegenden Textstellen in einen veränderten Kontext setzt und mit dionysischem Sinngehalt füllt.

Dionysische Kultelemente scheinen auch dort durch, wo Ampelos anderen Göttern gegenübergestellt wird, etwa bei einem Vergleich des Satyrn mit Apoll und Pan anlässlich des Syrinxspiels, das Ampelos für Dionysos zum Besten gibt (Nonn. D. 11,103112); der Vergleich im engeren Sinn findet sich in Nonn. D. 11,111f.:

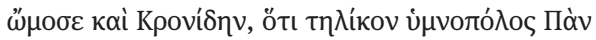

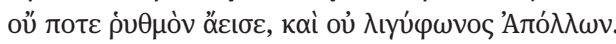

Er schwor auch beim Kroniden, dass auch der Hymnensänger Pan niemals einen solchen Rhythmus sang und auch nicht der hellstimmige Apoll.

Der Vergleichspunkt liegt in der Kunstfertigkeit der musischen Darbietung und zielt abermals auf Ampelos' Göttlichkeit ab, wenn Dionysos selbst seinen Schützling für fähiger als die eigentlich dafür zuständigen Götter hält. Auch dieser Vergleich ist eng an die dionysische Sphäre gebunden: Die Melodie, die Ampelos hier auf der Syrinx anstimmt (oúpı Ł̌v [Nonn. D. 11,104]), übertrifft sowohl die Hymnen des Pan als auch die Musik Apolls. Die neue, dionysische Kultmusik unterscheidet sich somit grundlegend von der bukolischen Hirtendichtung, für die Pan Sinnbild ist, sowie auch von geläufigen musischen Ausdrucksformen, repräsentiert durch den Gott Apoll mit „hell tönender Stimme“. ${ }^{9}$ Nonnos dient der Vergleich des Ampelos mit bereits etablierten

8 Vgl. h. Hom. 3 Ap. 134; Hes. Fr. 171,8 Merkelbach u. West; Pi. P. 3,14; Pi. I. 1,7, vgl. Chrétien (1985)

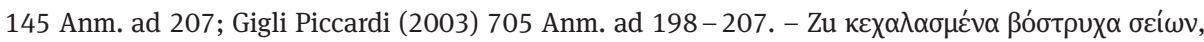
„die offenen Haarlocken schüttelnd“, vgl. Nonn. D. 4,137; 17,187; 18,144; 19,203; 31,3; 43,25f.; 46,8f.; 46,141.

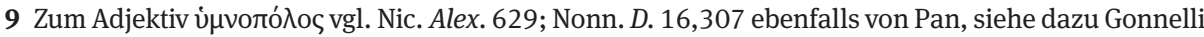

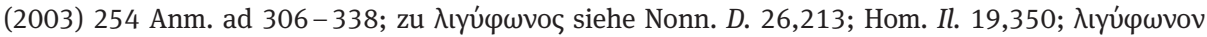


Göttern dazu, den neuen Gott Dionysos und seinen Kult gegenüber diesen aufzuwerten und den Protagonisten seines eigenen Epos, den Weingott, ins rechte Licht zu rücken.

Die Göttlichkeit des Satyrn wird insbesondere im Vergleich mit Gottheiten und Gestalten der Mythologie thematisiert, die eine besondere Affinität zu Licht und Glanz aufweisen. ${ }^{10}$ Ampelos wird je einmal mit der Mondgöttin Selene, dem Sonnengott Phaethon-Helios und Phosphoros, dem Morgenstern, verglichen. Das bereits aus der Eingangsszene um die im Fluss spielenden Satyrn (Nonn. D. 10,139-174) geläufige Glanz-Motiv wird im Folgenden zu einem Schlüsselmotiv. ${ }^{11}$ Die Licht- und Glanztopik nimmt Dionysos' Göttlichkeit ebenso vorweg wie die Satyrn, die zu seinem künftigen Kultpersonal gehören werden. Auch Ampelos wird mit göttlichem Glanz bedacht, und

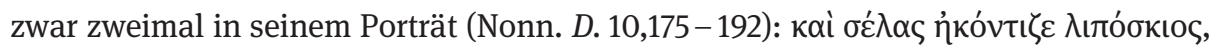

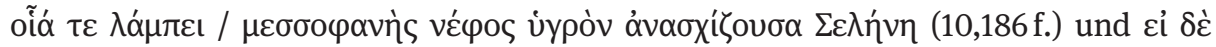

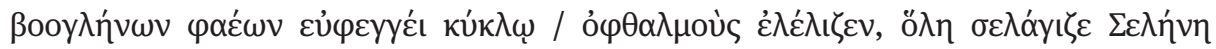
(191f.). Auffallend ist hier der mehrmalige Vergleich mit Selene. Nicht nur ihr Name fällt zweimal innerhalb von sechs Versen, Nonnos konstruiert ein regelrechtes ety-

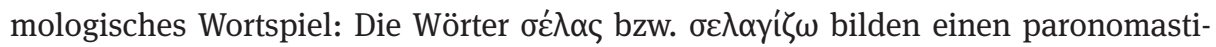
schen Gleichklang mit dem Namen $\Sigma \varepsilon \lambda \eta ́ v \eta .{ }^{12}$ Die Verknüpfung von Ampelos mit der

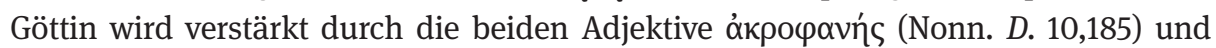

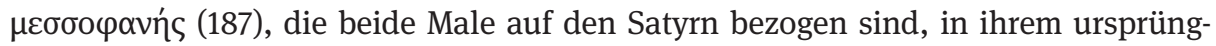
lichen Sinne jedoch die verschiedenen Mondphasen bezeichnen. ${ }^{13}$ Die Mondsichel

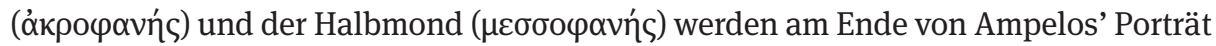

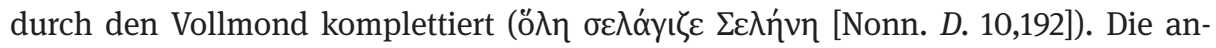
gedeuteten Phasen des Mondes verweisen auf das Wachstum der Weinpflanze, in die

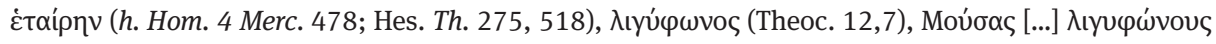

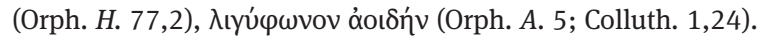

10 Farben-, Glanz- und Lichtmotivik sind auch wesentliche Elemente in der hellenistischen Dichtung, vgl. Hughes Fowler (1989) $17-20$.

11 Vgl. Vian (1995) 156.

12 Zu Nonnos’ Vorliebe für Etymologien vgl. Accorinti (1995-1996).

13 Vgl. Stegemann (1930) 39; Chrétien (1985) 144 Anm. ad 192. Das Adjektiv ớроџ

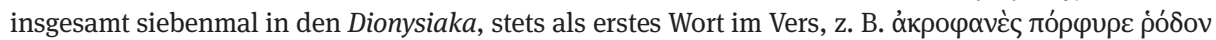

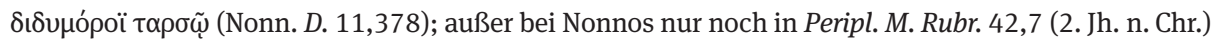
und Jo. Gaz. 1,278. - Beim Adjektiv $\mu \varepsilon \sigma \sigma o \varphi \alpha v n ́ \varsigma$ (,in der Mitte erscheinend“) handelt es sich um ein Wort, das ausschließlich bei Nonnos zu finden ist, 14-mal in den Dionysiaka (Nonn. D. 1,252; 2,636; 3,138; 5,184; 10,187, 412; 17,12; 18,112; 21,229, 284; 28,263; 33,75; 38,247; 43,285) und zweimal in der Paraphrase (Nonn. P. 6,7; 18,22). Am Beginn von Nonn. P. 6 setzt sich Jesus mitten unter seine

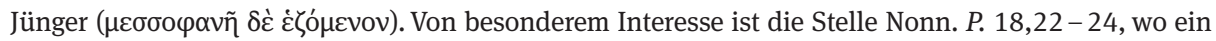
Windlicht eines der Verschwörer um Judas beschrieben wird; die Szene spielt in einem Jesus und den

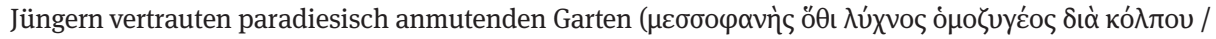

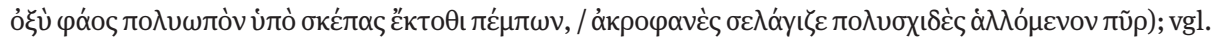
auch Nonn. P. 18,22 und 24; Livrea (1989) 120f. - Zum Bild des halb sichtbaren Mondes vgl. A. R. 4,1479f.; Verg. Aen. 6,453 f.; Hld. 5,8,5; Q. S. 1,147-149; zum zwischen den Wolken erscheinenden Mond vgl. Hom. Il. 8,555-559 und Q. S. 1,37-40. 
sich Ampelos verwandeln wird. ${ }^{14}$ Das zukünftige Geschehen wird einerseits durch das Glanzmotiv, das Ampelos erhält und das sein göttliches Wesen vermuten lässt, und andererseits durch die damit verbundene Göttin Selene vorweggenommen, deren Einwirken das Wachstum der Pflanzen lenkt.

In der Präsentation des Ampelos anlässlich seines ersten Auftritts findet sich nicht nur eine Reminiszenz an Apollonios Rhodios, sondern auch eine feine intertextuelle Anspielung auf einen Dichter, aus dem Nonnos im Verlauf der Dionysiaka immer wieder Wortmaterial schöpft und der eine bedeutende literarische Autorität für ihn darstellt: ${ }^{15}$ Der überaus hohe Stellenwert, den Nonnos Pindar einräumt, wird aus dem Binnenproömion der Dionysiaka am Beginn des 25. Buches ersichtlich, in dem er diesen, gemeinsam mit Homer, namentlich als sein Vorbild nennt (Nonn. D. 25,1-21, zu Pindar 11-21). ${ }^{16}$ Pindar ist für Nonnos der Dichter aus Theben, der spätantike Dichter bekennt sich selbst zur pindarischen Poetik (Nonn. D. 25,20 f.):

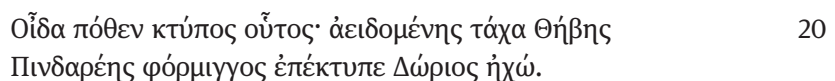

Ich weiß, woher dieser Klang kommt: Theben wird nun besungen, und aus pindarischer Phorminx erschallt eine dorische Weise.

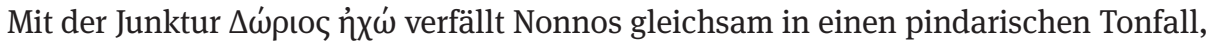
welcher es ihm erlaubt, einen Referenzautor in seine Dionysiaka aufzunehmen, der sich durch seine Dithyrambendichtung in der institutionalisierten kultischen Verehrung für Dionysos hervortat (Pi. Fr. 70a-88) und für alle folgenden Dichtergenerationen maßgeblich wurde. Pindar zählt in der Kaiserzeit genauso wie Homer, Euripides, Aristophanes und Theokrit zu den einflussreichsten griechischen Dichtern, deren Werke im höheren Schulunterricht verankert waren und deren profunde Kenntnis für Nonnos vorausgesetzt werden muss. ${ }^{17}$ Von besonderem Interesse für die Beziehung zwischen Nonnos und Pindar sind Pi. Fr. 70b, das mit der Nennung des Dionysos schließt und ursprünglich möglicherweise in die Erzählung der Geburt des Dionysos mündete, Pi. O. 2 und Pi. P.11, wo jeweils die Geburt Semeles geschildert wird, sowie Pi. N. 9 und Pi. Pae. 4 (= Pi. Fr. 52d), die das dionysische Getränk, den Wein, anführen. ${ }^{18}$ Die Ampelos-Episode verweist mit der Wahl ihrer poetischen Motive und mythischen Erzählungen immer wieder auf Pindars Dichtungen und rückt an einer Stelle den Dichter selbst ins Zentrum, so etwa in der Atropos-Rede, in der Ampelos

14 Vgl. Gigli Piccardi (2003) 701f. Anm. ad 181-187.

15 Zum Einfluss Pindars auf Nonnos siehe Haidacher (1949) 20 - 25; D’Ippolito (1964) bes. 69, 194, 196, 220, 240; Hernández de la Fuente (2008) 36; Gigli Piccardi (2016).

16 Zur Stelle vgl. Agosti (2004) 73; Bannert (2008).

17 Zum schulischen Autorenkanon in Ägypten in hellenistischer und römischer Zeit vgl. Cribiore (2001) 142, 201 - 204; zum Einfluss Pindars auf die griechische Rhetorik der Kaiserzeit vgl. Kennedy (1983) 49f.; Stenger (2008). Pindarisches findet ferner auch Eingang in die Werke der Dichter aus Gaza im 6. Jh. n. Chr., vgl. Ciccolella (2000) 124.

18 Vgl. Baeumer (2006) $39 \mathrm{f}$. 
zukünftiger Ruhm durch die hymnischen Darbietungen des Thebaners ihm zu Ehren prophezeit wird (Nonn. D. 12,147-153). ${ }^{19}$

Die Darstellung des Satyrn im 10. Buch der Dionysiaka ruft das Porträt des Kadmos aus der 4. Pythischen Ode Pindars in Erinnerung (Pi. P. 4,79-100). Der vor Pelias erstmals erscheinende Kadmos wird, ähnlich wie Ampelos, mit langen, glänzenden

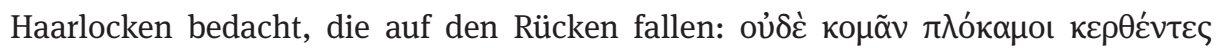

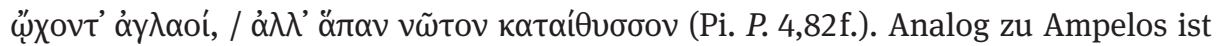
auch für Kadmos das Motiv Glanz gegenwärtig, außerdem lässt sich ein intertextueller Bezug zwischen den Dionysiaka und der pindarischen Ode festmachen: Das Verb

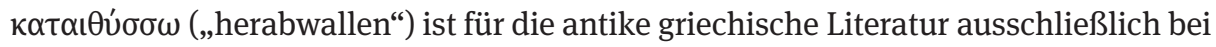
Pindar belegt (Pi. P. 4,83; 5,11), wovon in Nonn. D. 10,184 mit dem nonnianischen hapax legomenon ouvaı $\theta \dot{\sigma} \sigma \omega$ eine Variante geboten wird. ${ }^{20}$ Die Anknüpfung an Pindar erfolgt somit auf zwei Ebenen, über besonders distinguierte wörtliche Anklänge sowie über motivische Bezüge. An das Porträt des Kadmos schließt die Rede eines anonymen Beobachters an, der sich Fragen über die Identität des fremden Ankömmlings stellt (Pi. P. 4,86-100): Um welchen Gott oder Heros könnte es sich handeln? Etwa Apoll, Ares oder gar Otos, Ephialtes oder Tityos? Dasselbe rhetorische Schema bietet die DionysosRede in Nonn. D. 10,193-216. Zudem scheint auch bei Pindar eine Verbindung des Kadmos zur dionysischen Sphäre zu bestehen: Die Tatsache, dass Kadmos an der

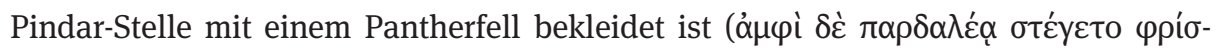

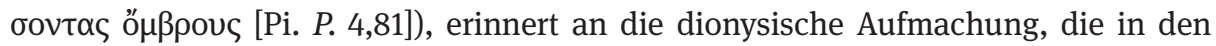
Dionysiaka allgegenwärtig ist und in der sich auch Ampelos zeigt (Nonn. D. 11,56-70,

19 Zur Rede der Atropos vgl. S. 167 f. - Ähnlich wie bei Nonnos wird auch für den Stil der pindarischen

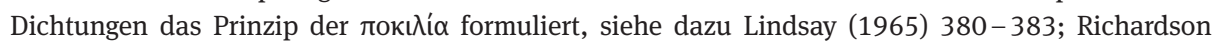
(1985) 385 f., 396 f.; García-Gasco (2007) 27 und Hernández de la Fuente (2008) 44. Bei Nonnos finden sich auch Themen und Motive, die aus der Dichtung Pindars geläufig sind, so etwa die Metapher des

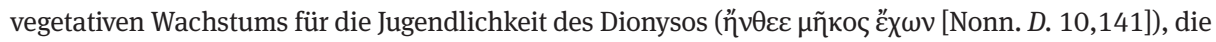

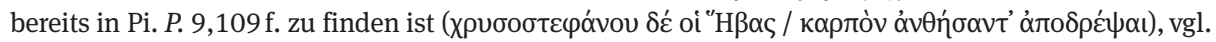
dazu auch Nonn. D. 15,170 und 204; die Horen als Göttinnen der Vegetation und des Wachstums (кaì

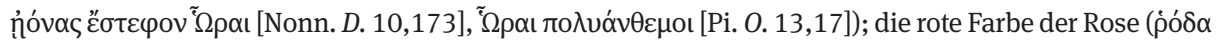

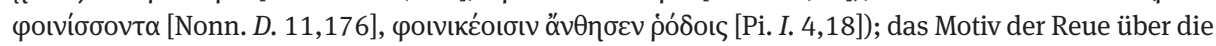
eigene Unsterblichkeit (Nonn. D. 11,325-327 für Dionysos und Ampelos und Pi. N. 10,75-85 für Kastor und Polydeukes); die sorgenlösende und therapeutische Wirkung des Weines (Nonn. D. 12,258 und Pi. Fr. 124,2 - 6 sowie Fr. 248; Nonn. D. 12,291 und Pi. Fr. 52d,25f.); für weitere mögliche An-

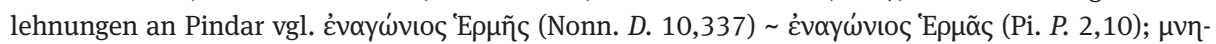

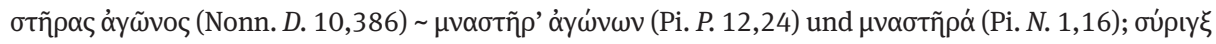

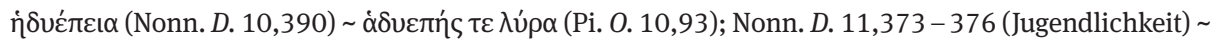

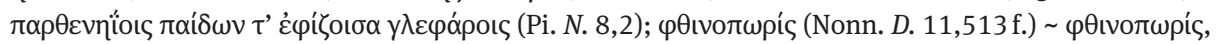

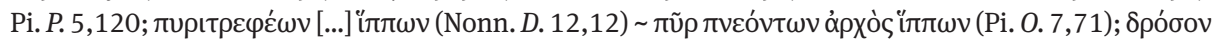

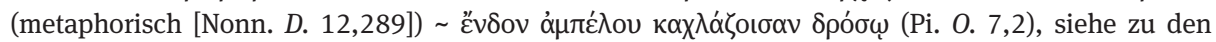
angegebenen Stellen in den Dionysiaka die Kommentare von Chrétien (1985) und Vian (1995) ad loc. 20 Vgl. Nonn. D. 43,295 und 346; zu aï ad loc. 
bes. 60 -63). Dionysos’ Großvater nimmt in den ersten Büchern der Dionysiaka eine prominente Rolle ein, der gemeinsame Kampf mit Zeus gegen Typhon, die Hochzeit mit Harmonia und seine Rolle als Kulturbringer in Griechenland bilden die Voraussetzungen für das Kommen des Weingottes.

Ein Nachhall pindarischer Dichtung findet sich möglicherweise auch im Vergleich mit dem Morgenstern, den Nonnos für Ampelos zieht und der gleichfalls in der 4. Isthmischen Ode Pindars auftaucht (Pi. I. 4,23f.): ${ }^{21}$

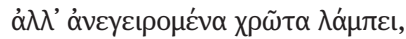

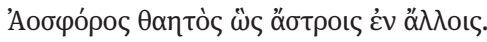

Aber erweckt strahlt sie an ihrem Leib, wunderbar anzusehen wie der Morgenstern unter den anderen Sternen.

Bei der Vergleichsstelle in den Dionysiaka handelt es sich um eine Passage in der Rede des Dionysos, in der er Ampelos zur Teilnahme am Schwimmwettbewerb auffordert und neben Phaethon-Helios auch Phosphoros, den Morgenstern, als göttliche Lichtgestalt benennt (Nonn. D. 11,22-27):

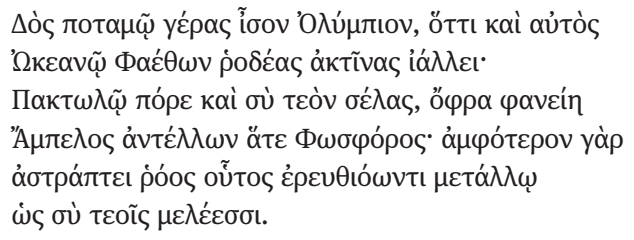

Gib dem Fluss ein Ehrengeschenk, das gleich einem olympischen ist, weil auch Phaethon selbst dem Okeanos rosenrote Strahlen entsendet. Dem Paktolos verleihe auch du deinen Glanz, damit (25) Ampelos wie der aufgehende Phosphoros erstrahlt. Denn beides glänzt, dieser Strom mit seinem rötlichen Metall wie auch du mit deinem Körper.

In der Pindar-Stelle übertrifft der Morgenstern sämtliche anderen Sterne. Vor diesem Hintergrund erfährt Ampelos in den Dionysiaka gleichsam einen vorzeitigen Katasterismos, eine verfrühte Vergöttlichung, da er, noch bevor ihn sein Todesschicksal ereilt, zum hellsten Stern von allen erklärt wird. Auch das inhaltliche Umfeld der Szene in der Ampelos-Episode lässt sich mit der Pindar-Ode in Verbindung bringen: Pindars Siegeslied auf den Athleten Melissos von Theben versetzt Ampelos ins Wettkampfmilieu. So wie bei Pindar der aufgehende Morgenstern zum Verkünder des sportlichen Sieges wird, fordert Dionysos zu Beginn des 11. Buches seinen Lieblingssatyrn zur Teilnahme am bevorstehenden Schwimmwettkampf auf und nimmt zugleich den Ausgang der Konkurrenz, den Sieg des Ampelos, vorweg.

Einen weiteren Hinweis darauf, dass Nonnos auf die Apotheose des Ampelos abzielt, liefert eine neuerliche intertextuelle Anspielung: Der Aufgang des neuen

21 Text: Snell u. Maehler (1980) 172; Übersetzung: Dönt (2007) 254f. Zum Vergleich der Stelle der Ampelos-Episode mit Pindar siehe Vian (1995) 156 ad loc. 
Sterns wird mit $\alpha \dot{\nu} \varepsilon^{\prime} \lambda \lambda \omega \nu$ markiert, einem Verb, das sich vor allem in der hellenistischen Literatur findet (vgl. A. R. 2,44; 3,520) und in astronomischen Kontexten den

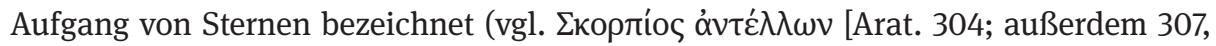
540 und 667]). ${ }^{22}$ Auch in den Dionysiaka wird das Wort im Zusammenhang mit astronomischen Inhalten verwendet (vgl. Nonn. D. 1,355 und 358; 38,85). Nonnos knüpft offenkundig an die mythische Tradition an, wie sie noch in den Fasten Ovids sichtbar wird, ${ }^{23}$ wonach Ampelos keine Metamorphose in einen Weinstock, sondern ein Katasterismos widerfährt. Nonnos geht es in der Ampelos-Episode nicht um die Konstruktion eines einzelnen, in sich geschlossenen Mythos, vielmehr bietet er ein möglichst breites Spektrum an Traditionen, Mythen und Motiven, sowie eine Formensprache, die den Anspruch erhebt, sämtliche Genera der griechischen Literatur aufzunehmen, darunter auch Stilelemente der pindarischen Epinikien und der hellenistischen Kleindichtungen.

In der Modellierung seiner Ampelos-Figur folgt Nonnos Pindar darin, dass er exklusives Wortmaterial sowie signifikante Inhalte übernimmt, um dies für seine eigene Poetik fruchtbar zu machen. Die Übernahmen stehen dabei vielfach im Dienste der Komposition. So laufen im Vergleich des Ampelos mit dem Morgenstern die kompositorischen Fäden zusammen: Während Dionysos in der Eingangsszene selbst durch die Fluten des Paktolos schwimmt, tut dies Ampelos am Beginn des 11. Buches, wo dieser zudem wiederholt mit seiner eigenen Göttlichkeit konfrontiert wird; seine

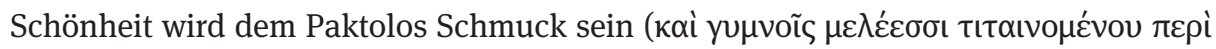

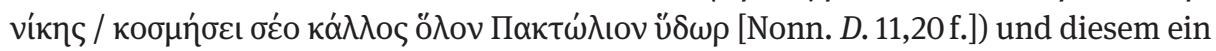
„göttliches Ehrengeschenk“ bereiten (22). Ähnlich wie in der Szene um das Bad des Dionysos in Nonn. D. 10,141-146 rührt der Glanz des Paktolos nicht nur von der Lichteinstrahlung und den darin befindlichen Goldpartikeln her, sondern auch vom überirdischen Glanz des Ampelos. Neben der Vorwegnahme von Ampelos' göttlichem Wesen verweist der Dichter in Nonn. D. 11,26f. auch auf das Kommen des Weinstocks: Das rötliche Metall des Flusses korrespondiert mit Ampelos' Gliedmaßen, den später Früchte tragenden Reben, und bereits in Nonn. D. 11,23 nimmt das Rot der über dem Okeanos aufgehenden Sonne das Rot des Weines und die Mischung von Wasser und Wein vorweg. Der Satyr Ampelos wird mit Göttlichkeit und mit dem Aussehen der neuen Pflanze in Zusammenhang gebracht, beides Eigenschaften, die auf das Ziel der Episode hinarbeiten: den göttlichen Status, den sich Dionysos erwerben muss, um Aufnahme im Olymp zu finden.

22 Vgl. Kröll (2013) 85 f.

$23 \mathrm{Zu}$ Ampelos bei Ovid vgl. S. 42. 


\subsection{Hyakinthos}

Als Vergleichsfiguren für Ampelos dienen vor allem jugendliche Göttergeliebte, die ebenfalls das Todesschicksal ereilt oder die eine besondere Verbindung mit ihrem Gott eingehen, sei es durch Metamorphose in eine Pflanze, die zu einem Attribut des Gottes wird, sei es durch die bloße Affinität zu einem signifikanten Wesensmerkmal des Gottes. Es handelt sich um ein gängiges literarisches Motiv, dessen sich bereits eine Reihe von Autoren vor Nonnos, insbesondere im Hellenismus, bedient hat. ${ }^{24}$ Die poetische Technik, die Nonnos in den Vergleichen anwendet, spiegelt zudem das rhetorische Genus der Synkrisis wider, die der Dichter ebenso wie andere rhetorische Kompositionsmittel souverän in seinem Epos zum Einsatz bringt. ${ }^{25}$

In den Büchern 10-12 wird Hyakinthos in seiner menschlichen oder auch pflanzlichen Erscheinungsform siebenmal zur Gegenüberstellung mit Ampelos herangezogen, zunächst in einer Szene im 10. Buch (Nonn. D. 10,250-255): ${ }^{26}$

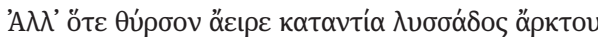
250

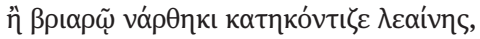

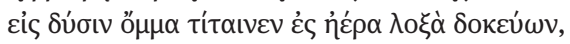

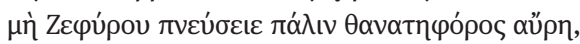

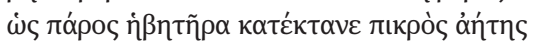

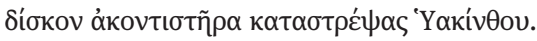

(250) Aber wenn er [sc. Ampelos] den Thyrsos gegen einen rasenden Bären erhob oder mit dem starken Narthexstengel auf eine Löwin schoss, richtete er [sc. Dionysos] seine Augen nach Westen und blickte zur Seite hin in die Luft, damit nicht wieder der todbringende Hauch des Zephyrs blase, wie schon einmal ein scharfer Windstoß einen Jüngling getötet hatte, (255) indem er eine Diskusscheibe als Wurfgeschoss auf Hyakinthos hinlenkte.

Nonnos orientiert sich an der Sagenversion, die auch Apollodor (Apollod. 1,3,3; 3,10,3) überliefert und die Ovid aufgreift (Ov. met. 10,162-219):: ${ }^{27}$ Die Konkurrenzsituation

24 Für die literarische Tradition und die ikonographischen Beispiele siehe Forbes Irving (1990) 129, 133; Calame (1999) 102-105, 160 - 164; Davidson (2007) 235-249.

25 Vgl. Kap. 6.3.

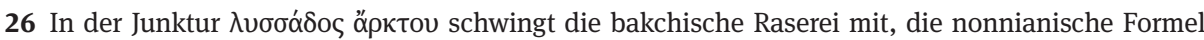
findet sich auch in Nonn. D. 2,619; 5,362; 9,195; 15,186; 24,136; 43,348; 44,30 und 48,251 wieder,

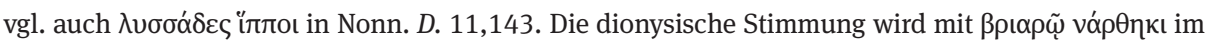

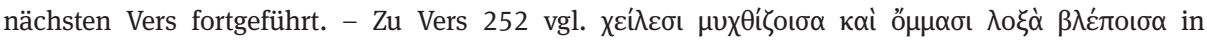

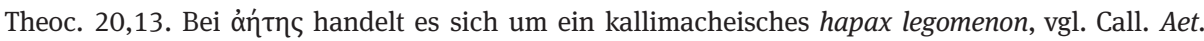
fr. 110,53 Pfeiffer (= 126,11 Asper), das dort zusammen mit dem Adjektiv $\theta \tilde{\eta} \lambda u \varsigma$ geboten wird und ebenfalls den Wind Zephyr bezeichnet, dieser „weibliche Wind“ steht für den Frühling und für

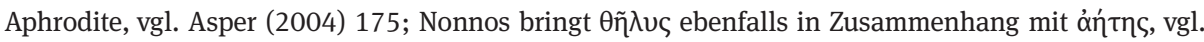
Nonn. D. 6,43 und 48,535, hier in der Ampelos-Episode setzt er in scharfem Kontrast zur Aitien-Stelle

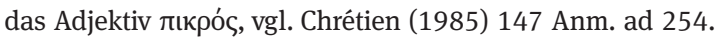

27 Vgl. Gigli Piccardi (2003) 294f. Anm. ad 153. Zu den literarischen und bildlichen Quellen des Hyakinthos-Mythos siehe Roscher Bd. I/2, 2759-2766; RE IX/1, 7-16; siehe außerdem Nic. Ther. 901 -906; Paus. 3,19,3; Philostr. Im. 1,24. 
zwischen Apoll und Zephyros führt zum Eklat, indem der von beiden geliebte Hyakinthos durch den von Apoll geschleuderten Diskus tödlich getroffen wird, weil Zephyros die Flugbahn beeinflusst hat. Auf die Klage Apolls folgt die Genese einer neuen Pflanze, die von nun an zum Symbol des Gottes wird. Mit der Einbettung dieses Mythos in die Ampelos-Episode nimmt Nonnos das Schicksal des Ampelos vorweg. Sein literarisch-mythologisches Spiel operiert mit dem Wissen des in den klassischen Mythen gebildeten Publikums, wobei es jedoch nicht bei einer Wiedererkennung von Bekanntem bleibt, sondern der Dichter über bloße Reproduktion von vertrauten Inhalten und Motiven hinausgeht und seinem Mythos eine Schlüsselfunktion für die Konzeption des Ampelos zuspricht. Mit dem Status des Götterlieblings, dem vorzeitigen Tod und der Wiederauferstehung in Gestalt einer Pflanze, einem dionysischen Kultsymbol, werden mehrere Elemente des Hyakinthos-Mythos auf Ampelos übertragen: Genauso wie Hyakinthos wird auch der junge Satyr sterben, um dann durch Metamorphose in eine Pflanze wieder zum Leben erweckt zu werden. Da die Gleichung sich auch auf Apoll und Dionysos übertragen lässt, erreicht Nonnos einen zweifachen Motivtransfer, in einen neuen Kontext und mit neuen Figuren. Traditionelle Motive werden auf neue Figuren übertragen, um deren göttlichen Status zu festigen.

Die Hauptvergleichspunkte zwischen Ampelos und Hyakinthos liegen auch in den übrigen Textstellen im frühen Tod und in der Verwandlung in eine Pflanze. ${ }^{28}$ In seiner ersten Klage auf den verstorbenen Ampelos stellt Dionysos sein eigenes Schicksal dem des Apoll gegenüber, wobei hier bereits von der Hyazinthe als Pflanze, nicht mehr vom Göttergeliebten die Rede ist (Nonn. D. 11,255-263). ${ }^{29}$ Dionysos nimmt das Schicksal des geliebten Satyrn vorweg, wenn er von der Zuordnung einer neuen Pflanze zu Apoll spricht, die durch die Metamorphose des Hyakinthos entsteht und dem Gott Trost über den schmerzlichen Verlust spendet. Mit einem weiteren traditionellen Motiv, den „neidischen Stieren“ (Nonn. D. 11,256), ${ }^{30}$ stellt Nonnos eine Verbindung zur Eifersucht des Zephyros im Hyakinthos-Mythos her: So wie Hyakinthos sein Leben verliert, wird Ampelos durch einen Stier ums Leben kommen. Das hellenistische Sujet wird in die Erzählung eingepasst und um ein neues, auf den unmittelbaren Kontext der Narration der Dionysiaka abgestimmtes Kompositionselement ergänzt: Zephyros scheint außer an dieser Stelle auch in der Erzählung um Kalamos und Karpos auf, wo als Grund für Karpos' vorzeitigen Tod ebenfalls der eifersüchtige Westwind in Erwägung gezogen wird (Nonn. D. 11,431, 455-457). ${ }^{31}$ Mittels wiederholter Anspielungen auf ein und dieselbe mythische Figur sowie motivischer Entsprechungen wird ein dichtes Netz an Referenzen geschaffen, das sich einerseits aus motivischen Vorbildern der hellenistischen Literatur, andererseits aus den Dionysiaka selbst zusammensetzt.

28 Vgl. Mazza (2012) $117 \mathrm{f}$.

29 Für Text und Übersetzung der Stelle siehe S. $126 \mathrm{f}$.

30 Die Version des Mythos, wonach Zephyros ebenfalls ein Werber um die Gunst des Hyakinthos gewesen sein soll, findet sich beispielsweise in Paus. 3,19,5.

$31 \mathrm{Zu}$ Kalamos und Karpos vgl. Kap. 7.2. 
Nonnos geht es darum, nicht nur das Schicksal des jungen Göttergeliebten zu thematisieren, sondern auch die Entwicklung des Dionysos, des Protagonisten der Dionysiaka, voranzutreiben. Die Vergleichsfiguren für den jungen Satyrn rühren an die Kernaussage der Ampelos-Episode: die literarische Inszenierung der Geburt des Weines und die Anerkennung des neuen Gottes. Neben dem Motiv des vor der Zeit verstorbenen Jünglings lässt der Dichter mit der Wendung åv $\theta \varepsilon \alpha \varphi \omega v \eta ́ \varepsilon v \tau \alpha$ in Nonn. $D$. 11,263 seine narrative Absicht durchscheinen, indem ein subtiler intratextueller Bezug zu Dionysos' zweiter Trauerrede hergestellt wird, die in Nonn. D. 11,316 die Junktur "A $\mu \pi \varepsilon \lambda о v ~ \alpha u ̉ \delta n ́ \varepsilon v \tau \alpha ~$ bietet. $^{32}$ Der direkte Bezug zu Ampelos und seine Verwandlung in einen Weinstock zeigt diesen zuallererst als Pflanze, die als Symbol und Wesensmerkmal für den neuen Gott vorgesehen ist, erst in zweiter Linie als eine autonome Figur.

Der Tod des Hyakinthos wird noch ein weiteres Mal zum Vergleich für das Schicksal eines Götterlieblings herangezogen (Nonn. D. 11,363-365), ${ }^{33}$ während Nonnos den Hyakinthos-Mythos an anderen Stellen der Episode dazu verwendet, um auf die Genese von Ampelos' Hauptattribut zu verweisen. Der dem bereits anerkannten und etablierten Apoll gegenübergestellte Dionysos wird zusätzlich zur Weinpflanze, ebenso wie Apoll, mit einem von seinem Attribut abgeleiteten Epitheton bedacht (Nonn. D. 11,329f.):

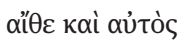

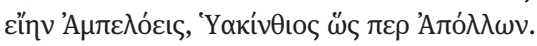

Beide Epitheta, Ampeloeis für Dionysos und Hyakinthios für Apoll, sind ausschließlich hier in den Dionysiaka belegt. ${ }^{34}$ Im Brennpunkt der Ampelos-Episode steht einmal mehr nicht Ampelos selbst, der nach seinem Tod vollständig im Weingott aufgeht, sondern Dionysos und seine Bewährung als Gott. Ebenfalls auf den in eine Pflanze verwandelten Hyakinthos wird in zwei Szenen im 12. Buch verwiesen: In der Rede der Atropos, die der Metamorphose unmittelbar vorausgeht, prophezeit die Moire Dio-

32 Vgl. Gigli Piccardi (2003) 778 Anm. ad 255-263. Ein Detail zum Verständnis nonnianischer

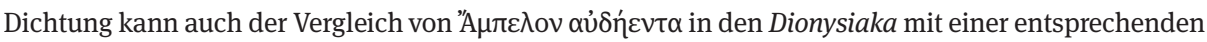

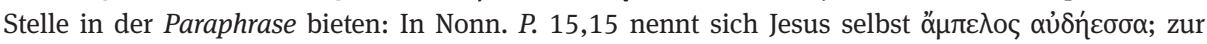
Bedeutung der Wortverbindung in der Paraphrase siehe Caprara (2008) und Spanoudakis (2014b) 294f. Anm. ad 169a.

33 Hier will Eros am Beginn seiner Rede anhand von mythologischen Beispielen Dionysos demonstrieren, dass sein Liebeskummer nur durch eine neue Liebe zu überwinden sei; Dionysos solle sich am Windgott Zephyros ein Beispiel nehmen, der sich nach dem Verlust des Hyakinthos den Jüngling

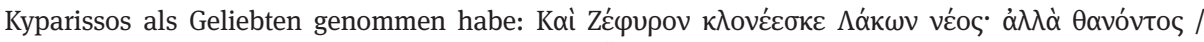

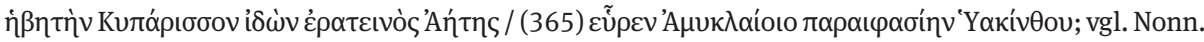
D. $11,356-362$.

34 Vgl. Del Corno u. a. (1997) 316; Gigli Piccardi (2003) 785 Anm. ad 328-330. - Für Zeus ist mit Ampeleites ein vergleichbares Epitheton belegt, vgl. dazu Kap. 3, Anm. 14. 
nysos sein künftiges Attribut, mit dem er sogar Apoll und seine Hyazinthe übertreffen werde (Nonn. D. 12,154-157)..$^{35}$ Die Genese des neuen Attributs für Dionysos ist mit einer Aufwertung des neuen Gottes verknüpft. Das Motiv des Neides, welches bereits im Zusammenhang mit Zephyros begegnet ist (Nonn. D. 11,255-263), wird nun auf Apoll übertragen; so wie im traditionellen Mythos Zephyros wegen des schönen Hyakinthos voll von Neid auf Apoll ist, wird Apoll nun seinerseits Eifersucht gegen Dionysos und seinen Weinlaubkranz hegen. In der die Ampelos-Episode beschließenden Rede des Dionysos erfolgt die Synkrisis des Weinstocks und der Hyazinthe Apolls mit dem Fazit, dass die neue Pflanze in allen Punkten die vom Mythos bekannte übertreffe (Nonn. D. 12,207-209, 237-241). ${ }^{36}$ Die Vergleiche sind nicht nur nach dem rhetorischen Muster der Synkrisis gearbeitet, Nonnos knüpft auch an Stilelemente, Motive und Topoi der hellenistischen Literatur an, ${ }^{37}$ so hat etwa bei Theokrit die Braut Helena ihr Haar mit Hyazinthen geschmückt (Theoc. 18,2). ${ }^{38}$ Auch die insbesondere im Hellenismus beliebte poetische Technik des Katalogs wird von Nonnos in einigen der zitierten Passagen angewandt: Der Blumenkatalog in Nonn. D. 12,237-241 hat seine Musterbilder in hellenistischen Pendants, wie sie etwa bei Moschos zu finden sind (Mosch. II,63-71 Beckby; Mosch. III,3-8 Beckby; siehe außerdem AP 4,1 und 2; 5,147), ebenso zählen die Kataloge sterblicher Götterlieblinge zum Standardrepertoire griechischer Dichtung, etwa ein Katalog von jungen, männlichen Göttergeliebten, darunter auch Hyakinthos, in AP 12,128. ${ }^{39}$

Nonnos' Auseinandersetzung mit der hellenistischen Dichtung manifestiert sich an einem regelrechten Motivnetz, das der Dichter für Ampelos aufspannt und in das er die zahlreichen Vergleichsfiguren mühelos integriert. Nicht nur der Typus des jugendlichen Göttergeliebten, sondern sämtliche damit verbundenen Teilmotive werden von ihm auf Ampelos übertragen: ${ }^{40}$ der verfrühte Tod, die Trauer des Gottes und der Natur, der Trost durch eine neue, durch Metamorphose geschaffene Pflanze sowie deren Verbindung zum Kult des Gottes. Die Parallelisierung mit Hyakinthos und anderen mythischen Figuren mit vergleichbarem Schicksal steuert die Rezeption der Erzählung und dient als Lesehilfe. Dieser wiederkehrende Charaktertypus hat mehrmals Präzedenzfälle in der hellenistischen Dichtung, etwa in der Bukolik Bions, der

35 Zu Hyakinthos an dieser Stelle vgl. D’Ippolito (1964) 145.

$36 \mathrm{Zu}$ Text und Übersetzung beider Stellen siehe S. 169-172.

37 Zu Nonnos' Verarbeitung hellenistischer Quellen in der Ampelos-Episode siehe Kap. 5.3. - Die Hyazinthe als Pflanze samt ihrer Symbolik der Göttlichkeit und Schönheit begegnet bereits bei Homer: In Hom. Il. 14,348 sprießt sie zusammen mit anderen Blumen anlässlich des ícpò yó $\mu$ oৎ von Zeus und Hera und markiert das unmittelbare Geschehen als göttlich. In der Odyssee erhält Odysseus durch göttliche Einwirkung Athenes ein außergewöhnlich schönes Äußeres, sein Haar fällt vergleichbar mit Hyazinthenblüten auf die Schultern herab (Hom. Od. 6,231; 23,158).

$38 \mathrm{Zu}$ Hyazinthen bei Theokrit siehe ferner Theoc. 10,28; 11,26.

39 Vgl. auch Bion III,12 (Beckby). - Auch die spätantike rhetorische Prosa bedient sich dieses Stilelements, vgl. Procop. Gaz. Op. I [= Dial. 1],2 mit der Aufzählung von Pan und Echo, Apoll und Hyakinthos sowie Narkissos.

40 Zum Hyakinthos-Mythos in der Ampelos-Episode vgl. Mazza (2012) 117 - 119. 
sämtliche Motive vereint, die auch Nonnos für Ampelos formuliert. Eine Stelle bei Bion ist für das Verhältnis zwischen Hyakinthos und Ampelos besonders aufschlussreich: Im Gedicht Bion III,1 (Beckby) befindet sich Apoll in derselben Situation wie Dionysos in Nonn. D. 12,117-137, beide Gottheiten trauern um ihre verlorenen Geliebten und finden Trost in einer Pflanze, die durch Metamorphose der einstigen Jünglinge entsteht, welchen gleichsam eine Apotheose zuteil wird, indem sie eng an den Gott und seinen Kult gebunden werden.

Die Figur des Hyakinthos wird nicht nur zur Modellierung des sonst kaum bekannten Ampelos herangezogen, sondern zieht auch wichtige Kompositionslinien. Hyakinthos wird viermal vor und elfmal nach der Ampelos-Episode genannt, wobei er davor ausschließlich in seiner pflanzlichen Gestalt und stets in Verbindung mit Apoll erscheint: In der Auseinandersetzung zwischen Typhon und Zeus im 2. Buch trauert Apoll um die vernichtete Vegetation, insbesondere um seine Hyazinthe, ${ }^{41}$ Hyazinthen wachsen im Garten des Emathion auf Samothrake, ${ }^{42}$ als Pflanze des Apoll werden sie auch im Porträt des Kadmos genannt. ${ }^{43}$ Durch die motivischen Entsprechungen schlägt der Dichter eine Brücke zwischen Kadmos und Dionysos, die eine direkte Verbindung der beiden mythischen Generationen schafft. Das frühe Auftauchen der symbolhaften Pflanze in den Dionysiaka nimmt die Ankunft des Protagonisten Dionysos vorweg und verleiht der mythischen Genealogie des Weingottes zusätzliche Glaubwürdigkeit.

Von den elf Textstellen nach der Ampelos-Episode, an denen von Hyakinthos oder der Hyazinthe die Rede ist, ${ }^{44}$ sind besonders diejenigen von Interesse, welche die Personifikation des Jünglings bieten. Von Auferstehung ist im lediglich zwei Verse umfassenden Lied des Oiagros im Rahmen der Leichenspiele zu Ehren des Staphylos die Rede (Nonn. D. 19,104f.):

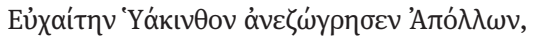

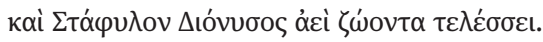

105

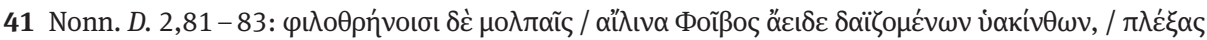
$\pi \varepsilon ́ v \theta \mu$ ำ Hyazinthen und flocht einen kummervollen Lobgesang“.

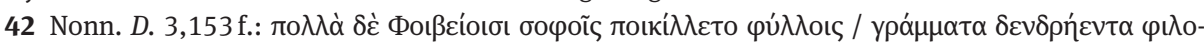

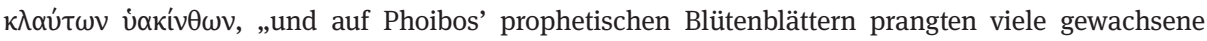

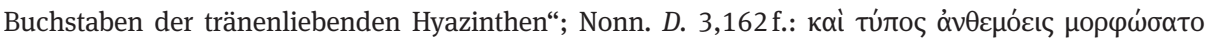

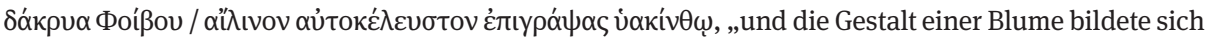
aus Phoibos' Tränen und schrieb von selbst ein Klagelied auf die Hyazinthe“.

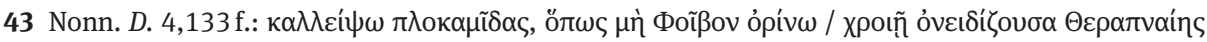

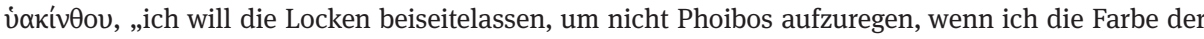
therapnäischen Hyazinthe tadle“; zum Porträt des Kadmos vgl. Chuvin (1976) $64 \mathrm{f}$.

44 Nonn. D. 16,117 (Hymnos an Nikaia); 18,75-77 (die Hyazinthe als Schmuck von Staphylos' Palast); 19,104f., 187 f.; 29,95-99, 142; 32,27; 33,131-133; 40,256f. (die Hyazinthe als Kriegsbeute des dionysischen Heeres); 42,294-300; 48,581-589. 
Den schönhaarigen Hyakinthos belebte Apoll wieder, (105) und dem Staphylos wird Dionysos ewiges Leben bereiten.

Hyakinthos verbindet auch Ampelos und Hymenaios, welchem ein dem Ampelos ähnliches Schicksal beschieden ist (Nonn. D. 29,95-99): ${ }^{45}$

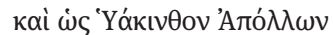

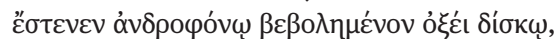

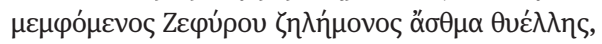

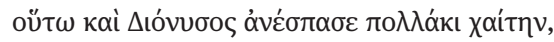

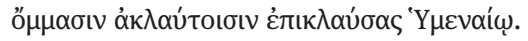

95

(95) Und wie Apoll Hyakinthos betrauerte, als er getroffen war vom scharfen, männermordenden Diskos, und die Sturmböe des neidischen Zephyros tadelte, so raufte sich auch Dionysos oft das Haar und beweinte mit tränenlosen Augen Hymenaios.

In den abschließenden Büchern des Epos wird die Hyazinthe in dionysischem Kontext sichtbar: In seiner Rede an Beroe prophezeit Dionysos die Ankunft des Weines (Nonn. D. 42,294-300), indem er innerhalb weniger Verse das ganze Spektrum an dionysi-

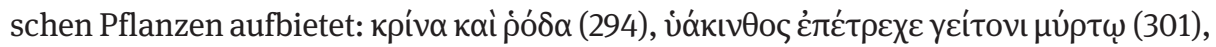

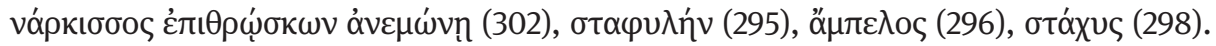
Im Anschluss daran schildert der sich als Landmann ausgebende Dionysos seine Tätigkeiten, beginnend und endend mit der Kultivierung des Rebstocks (42,303-312). Ebenso ist in der Ekphrasis der Quelle der Aura die Hyazinthe mit der dionysischen Sphäre verbunden (48,571-589): Der locus amoenus ist durch eine Weinquelle markiert, die Dionysos eigenhändig unter Zuhilfenahme eines Thyrsos sprudeln lässt, ferner durch die Horen, die die Felsabhänge mit Blumen schmücken, sowie durch

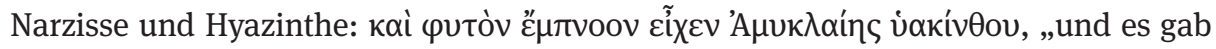
die beseelte Pflanze der amykläischen Hyazinthe“ (587).

Nonnos verfolgt mit dem Hyakinthos-Mythos als narratives Ziel die Vorbereitung auf den Weingott, der als Gott der vegetativen Sphäre periodisch stirbt und wieder ins Leben gerufen wird, er knüpft an einen aus der literarischen Tradition geläufigen Mythos an und übernimmt dessen Kernelemente, um daraus seinen eigenen Mythos zu schaffen. Diese neue Erzählung ist nicht ausschließlich aus sich selbst heraus verstehund erfassbar, sondern konstituiert sich vielmehr aus einem kontinuierlichen Dialog mit literarischen Vorbildern und mythologischen Folien und setzt Hyakinthos als Chiffre für Tod und Wiedergeburt. Das spätantike Epos unterscheidet sich insofern vom klassischen, als es die eigentliche epische Handlung über weite Strecken auf Vergleiche und erzählte Bilder projiziert, die per se keine direkten Verbindungen zur Haupterzähllinie aufweisen, deren Verlauf jedoch in hohem Maße bestimmen.

45 Vgl. auch die daran anschließende Rede des Dionysos auf den verwundeten Hymenaios: Фoĩßov हैa

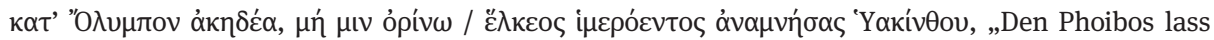
unbekümmert im Olymp, damit ich ihn nicht aufrege, wenn ich ihn an die Wunde des lieblichen Hyakinthos erinnere“ (Nonn. D. 29,142f.). 


\subsection{Narkissos}

Ähnlich wie der Mythos um Hyakinthos ist auch der Narkissos-Mythos mit der Genese einer Pflanze verbunden, beide werden in der vom Dichter ausführlich diskutierten Aura-Episode genannt (Nonn. D. 48,581-586): $:^{46}$

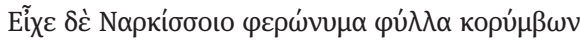

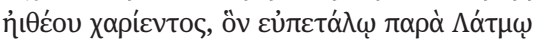

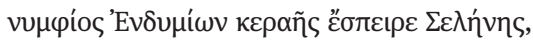

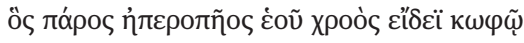

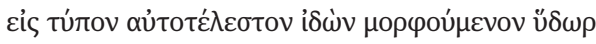

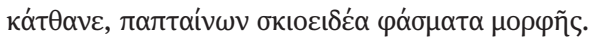

Und er hatte Efeutrauben, die den Namen des Narkissos trugen, des lieblichen Jünglings, den am Latmos, dem schönbelaubten, Endymion als Bräutigam der gehörnten Selene zeugte: Der sah einst, wie im trüben Bild seines trügerischen Körpers (585) das Wasser sich von selbst in das Abbild verwandelte, und starb, als er die schattigen Schemen seiner Gestalt erblickte.

Der nur auf den ersten Blick idyllische Ort erhält durch die Verbindung mit den Pflanzen einen zusätzlichen Bedeutungshorizont: Durch das Aufgreifen des Todesschicksals der beiden jungen Männer wird Auras eigenes Schicksal vorskizziert, eine narrative Technik, die Nonnos auch in der Konzeption seiner Ampelos-Figur anwendet. Der bereits in die Pflanze verwandelte Narkissos wird dreimal dem Satyrn gegenübergestellt, zunächst vor Beginn der Wettkämpfe zwischen Dionysos und Ampelos, als sich Eros einen Kranz aus Narzissen und Hyazinthen flicht (Nonn. D. 10,336338): ${ }^{47}$

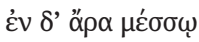

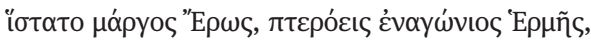

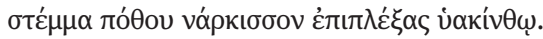

Und in der Mitte stand der rasende Eros als geflügelter und kämpferischer Hermes, der als Kranz des Verlangens eine Narzisse mit einer Hyazinthe verflochten hatte.

Der Blumenschmuck hat zweifache Funktion: Er enthält zum einen - insbesondere durch die Anwesenheit des Eros - eine erotische Komponente und weist den Satyrn als jungen Göttergeliebten aus, zum anderen verweist er auf den Stier als zukünftiges Opfertier im Rahmen des dionysischen Kultes und somit auf Ampelos’ Tod. ${ }^{48}$ Dieser Bezug wird insbesondere aus zwei weiteren Stellen ersichtlich: Die Narzisse wird von Ampelos bei der zunächst friedlichen Konfrontation mit dem Stier ebenfalls als Schmuck verwendet (Nonn. D. 11,175-178), indem der Satyr den Stier mit frischem

46 Zur Stelle siehe Accorinti (2004) 693-695 Anm. ad 581-586. - Für die antiken literarischen Quellen zum Narkissos-Mythos siehe Roscher Bd. III/1, 10-21; Bettini u. Pellizer (2003) $43-114$. 47 Vgl. Chrétien (1985) 153 Anm. ad 138.

48 Vgl. Del Corno u. a. (1997) 314; Gigli Piccardi (2003) 768f. Anm. ad 175-178. 


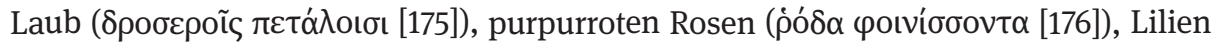

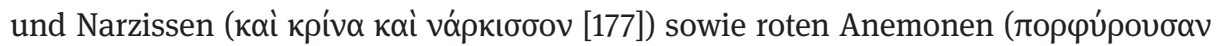

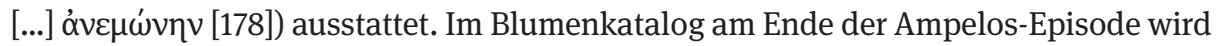
die Narzisse in Gesellschaft derselben Pflanzen genannt, ergänzt um Weinstock und Traube (Nonn. D. 12,337-341)..$^{49}$ Der Bezug zwischen Narzisse und Tod wird ferner aus einer Stelle bei Theokrit deutlich, in der beim Tod des Daphnis mehrere Pflanzen, darunter auch die Narzisse, aufgezählt werden (Theoc. 1,132-136).

Neben der Narzisse als Ergebnis der Verwandlung des Knaben ist in der AmpelosEpisode auch der personifizierte Narkissos zu finden, der zunächst in der ersten Dionysos-Rede Ampelos zur Seite gestellt wird (Nonn. D. 10,214-216):

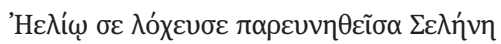

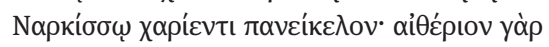

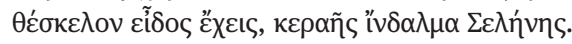

Dem Helios hat dich Selene geboren, als sie bei ihm lag, (215) dich, der du dem lieblichen Narkissos in Allem gleichst. Denn eine himmlische, wunderbare Gestalt hast du, ein Abbild der gehörnten Selene.

Die Synthese von Narkissos und Selene sowie deren Bezug auf Ampelos kehren dessen Göttlichkeit hervor und implizieren eine Apotheose des Satyrn. ${ }^{50}$ Auch in seiner zweiten Klagerede auf Ampelos vergleicht Dionysos diesen mit Narkissos. In einer von Dionysos selbst geäußerten fingierten Rede des Zeus an ihn, Dionysos, sucht er Linderung seines Schmerzes (Nonn. D. 11,321-324):

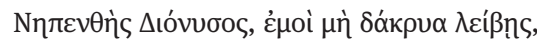

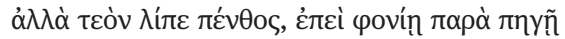

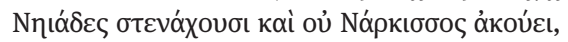

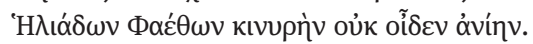

Sorgenvertreibender Dionysos, vergieße mir keine Tränen, sondern lass ab von deinem Leid, weil bei der tödlichen Quelle auch die Najaden aufseufzen, Narkissos sie nicht hört und Phaethon nichts von der traurigen Klage der Heliaden weiß!

49 Nonnos setzt Blumenkataloge in den Dionysiaka häufiger ein, vgl. Vian (1995) 164f., er orientiert sich dabei an hellenistischen Vorbildern wie etwa Mosch. II,63-71 Beckby, wo Europa und ihre Begleiterinnen sich an verschiedenen Blumen erfreuen, unter anderem an Hyazinthen und Narzissen. Vergleichbare Kataloge u. a. mit Narzissen finden sich in Gedichten der griechischen Anthologie: AP 4,1 (Kranz des Meleagros); 5,74; 5,144 und 147; 11,34; zu Narkissos außerdem AP 11,76 und Claud. rapt. Pros. 2,128-136 (vgl. die zu den Hyazinthen und Narzissen zugehörigen Mythen). - Der Verweis von D’Ippolito (1991) 529f. auf mögliche lateinische Quellen für das Schmücken des Stieres (vgl. Silvia in Verg. Aen. 7,488 und Kyparissos in Ov. met. 10,123 - 125) kann aufgrund der zahlreichen Beispiele aus der griechischen Literatur nicht überzeugen.

50 Vgl. Gigli Piccardi (2003) 705 f. Anm. ad 214-216. 
Die Motive Schönheit, Liebe, Tod und Aition sind für die Konstitution der Mythen um Narkissos und Hyakinthos entscheidend. ${ }^{51}$ Der mythisch-literarischen Überlieferung zufolge lässt sich zudem eine Verbindungslinie zwischen Narkissos und Dionysos ziehen:52 So entwirft der Chor im 3. Stasimon des sophokleischen Ödipus auf Kolonos einen locus amoenus des Haines von Kolonos (S. OC 668-719) und präsentiert die blühende Vegetation mit Efeu, Wein, Narzisse und Krokus sowie den Kult des Dionysos als charakteristisches Element der Landschaft. ${ }^{53}$ Ein dem Narkissos-Mythos eigentümlicher Aspekt stellt eine weitere Verbindung zum Dionysischen her: Das Motiv des Spiegelbildes, das mit Narkissos verbunden ist, wird vom Dichter auch an einer Schlüsselstelle der Dionysiaka eingesetzt; genauso wie Narkissos am eigenen Spiegelbild scheitert, stirbt Zagreus, der erste Dionysos, unter Einwirkung der Titanen durch den Blick in den Spiegel (Nonn. D. 6,169-173), beide entstehen jedoch neu in einer jeweils anderen Form, Narkissos wird zur Pflanze, Zagreus wird in Gestalt des Weingottes ähnlich wie auch Ampelos wiedergeboren. ${ }^{54}$

Der Plan, den Nonnos mit der Wahl des Narkissos als Vergleichsfigur für Ampelos verfolgt, wird durch den Blick auf die weiteren Stellen vervollständigt, an denen die Narzisse und Narkissos in den Dionysiaka in Erscheinung treten: Im 15. Buch wird

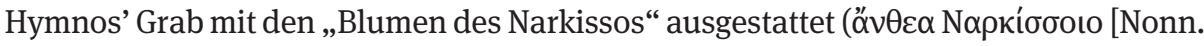
D. 15,353]), beim ícò ү yó $\mu$ os von Zeus und Hera zeichnen die sich umschlingenden Anemonen und Narzissen die fruchtbare Verbindung der beiden Götter nach (32,91f.), und schließlich erhält im Pflanzenkatalog, den Dionysos für Beroe zum Besten gibt, der neue Weinstock den Vorzug gegenüber anderen Gewächsen (42,294-300, bes.

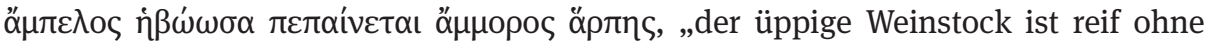
Sichel“ [296]).

51 Abermals orientiert sich Nonnos nicht nur an den Eckpunkten, die in sämtlichen literarischen Zeugnissen zu Narkissos überliefert sind, sondern bietet auch unkonventionelle Aspekte: Ein Nachhall der in der Anthologia Latina tradierten Version, wonach Narkissos auf der Suche nach seinem Vater den Tod im Fluss fand ( $A L 210$ Shackleton Bailey sowie AL 259 und 260 [Pentadius] Shackleton Bailey), findet sich im Schwimmwettkampf zwischen Kalamos und Karpos; auf das Motiv des Todes durch den eigenen Vater, einen Flussgott, spielt Kalamos in Nonn. D. 11,431-438 an, wenn er, der Sohn des Flussgottes Maiandros, über den Verbleib seines geliebten Karpos rätselt, der soeben in den Fluten

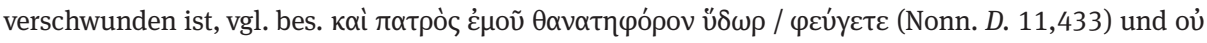

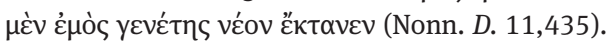

52 Vgl. Bettini u. Pellizer (2003) 89, 98, 102.

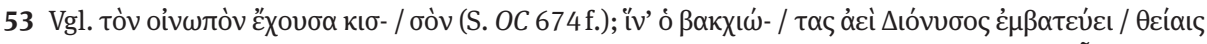

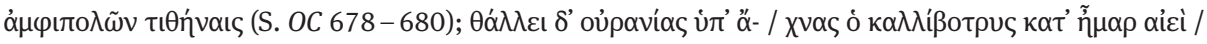

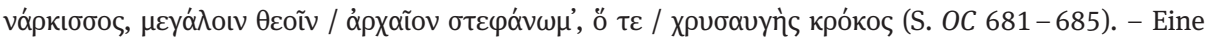
Gemeinsamkeit zwischen Narzisse und Wein liegt zudem in der antiken Vorstellung, wonach beide Gewächse über eine narkotische Wirkung verfügen und Benommenheit hervorrufen können; vgl. Bettini u. Pellizer (2003) 79f., 154; Gigli Piccardi (2003) 706 Anm. ad 214-216.

54 Spiegel und Spiegelbild gelten im spätantiken Neuplatonismus als Symbol des irdischen Daseins. Die Betrachtung im Spiegel steht symbolisch für die Verhaftung der menschlichen Seele in der irdischen Welt, vgl. Plot. Ennead. 1,6,8. 


\subsection{Pelops}

Nonnos wendet sich an ein Publikum, das tief im mythologischen Kosmos der Antike verwurzelt und nicht nur imstande ist, signifikante Szenen, Motive und Figurentypen, sondern auch Elemente der sprachlichen Mikroebene zu identifizieren und in der literarischen Tradition zu verorten. Um die Unsterblichkeit des Satyrn herauszuarbeiten und zu begründen, bedient er sich des Pelops-Mythos in Pindars 1. Olympischer Ode, dem er auch im Gebrauch der Lexik folgt. Zweimal erscheint Pelops in den Dionysiaka als Musterbild für Ampelos (Nonn. D. 10,260-263; 11,271-275): $:^{55}$

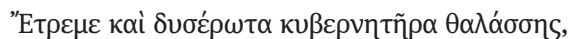
260

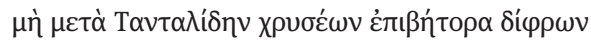

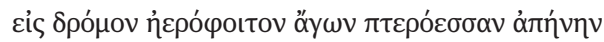

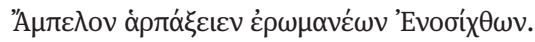

(260) Er zitterte auch vor dem Steuermann des Meeres in seinem Liebeswahn, dass er nach dem Tantaliden, der auf den goldenen Wagen stieg, sein geflügeltes Gefährt auf den luftigen Pfad lenken und auch Ampelos rauben könnte, der Erderschütterer, rasend vor Liebe.

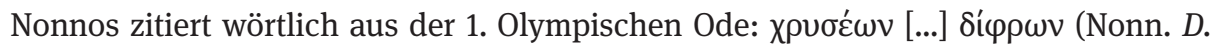

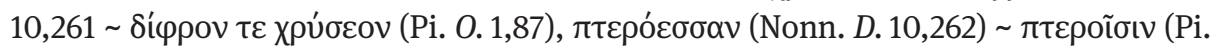

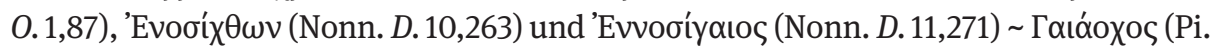

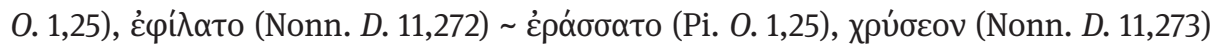

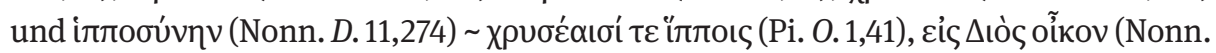

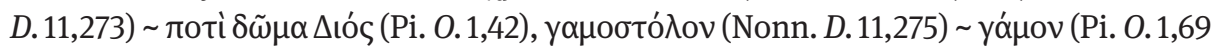

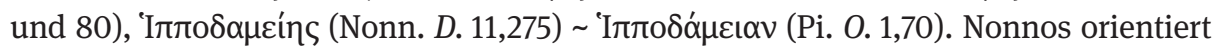
sich auch hinsichtlich des Inhalts genau an seinem Vorbild und greift mit der Liebe des Gottes Poseidon zu Pelops, der Entführung in den Olymp sowie dem Wagenrennen um die Gunst Hippodameias die drei Hauptmotive des Mythos auf. ${ }^{56}$ Das Schicksal des Ampelos rückt nahe an das des Pelops: Genauso wie Pelops von Poseidon in den Olymp entrückt wurde, wird Ampelos Unsterblichkeit erlangen. Als Vergleichspunkt dient zudem der Wettkampf-Kontext des Pelops-Mythos: Der durch göttliche Hilfe errungene Sieg des Pelops im Wagenrennen gegen Oinomaos entspricht der Überlegenheit des Ampelos in den drei sportlichen Agonen, die ebenfalls ausschließlich durch den Willen eines Gottes erreicht wird. Auch die negative Seite des Pelops, die Freveltat, die Tantalos an seinem Sohn verübt, sowie dessen Wiederbelebung durch

55 Für Text und Übersetzung der Stelle Nonn. D. 11,271 - 275 siehe S. 126f. - Pelops wird in den Dionysiaka noch an anderen Stellen erwähnt: als eponymer Heros der Peloponnes (Nonn. D. 3,259), als Jüngling mit der Elfenbeinschulter (Nonn. D. 18,27), als Wagenlenker (Nonn. D. 37,137 und 308) und als Gatte der Hippodameia (Nonn. D. 11,275; 20,157-165; 33,292-296; 37,338-341; 48,212 - 214), vgl. Del Corno u. a. (1997) 306; Chrétien (1985) 148 Anm. ad 263; Gigli Piccardi (2003) 710 Anm. ad 260-263, 779f. Anm. ad 271-275. - Zum Pelops-Mythos bei Pindar vgl. Davidson (2007) 221-227.

$56 \mathrm{Zu}$ den inhaltlichen Entsprechungen vgl. Vian (1995) 171 Anm. ad 271-275. 
die Götter werden im Ampelos-Mythos wieder aufgenommen. Die Zerstückelung des Pelops und die anschließende Wiedererweckung durch die Götter spiegeln sich in der Zagreus-Erzählung im 6. Buch der Dionysiaka sowie in Ampelos' Tod und Wiedergeburt in den Büchern 10-12.

Nonnos lehnt sich an literarisch-mythische Musterbilder an, er übernimmt ausgewählte sprachliche Komponenten ebenso wie geläufige Motive und größere Rahmenhandlungen, imitiert jedoch nie bedingungslos, sondern verfolgt stets eigene Handlungsziele. Den Typus des männlichen Göttergeliebten, der als narrative Folie für Ampelos dient, schöpft Nonnos nicht nur aus der hellenistischen Dichtung, sondern auch aus den Pindarischen Oden. Von den drei Jünglingen des Katalogs in Nonn. $D$. 10,250 - 263 finden sich mit Pelops und Ganymed zwei an einer Pindar-Stelle wieder (Pi. 0. 1,36-45). ${ }^{57}$ Insbesondere mit der Figur des Ganymed verweist der Dichter nachdrücklich auf seinen Ampelos-Mythos und auf dessen Ergebnis, den Wein.

\subsection{Ganymed}

Mit der Aufnahme der Figur des Ganymed in seinen mythologischen Kosmos bedient sich Nonnos einer langen literarischen Tradition, die bis zur homerischen Ilias zu-

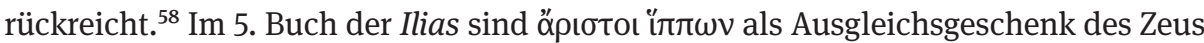
an Ganymeds Vater für den Verlust seines Sohnes genannt (Hom. Il. 5,265-267,

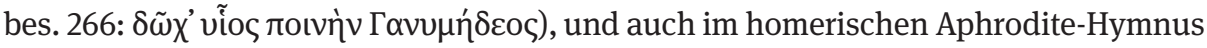
lässt der Göttervater Pferde zum Trost zurück, nachdem er den schönen Ganymed geraubt und als Mundschenk der Götter in den Olymp gebracht hat (h. Hom. 5

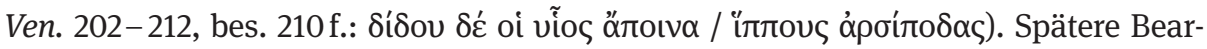
beitungen setzen neue Akzente, vor allem im Hellenismus wird die Darstellung des Liebesverhältnisses zwischen Zeus und Ganymed und eine damit verbundene erotische Konnotation betont, ${ }^{59}$ weitere Themen sind der Aufstieg zu den Göttern und die Einsetzung Ganymeds als Mundschenk.

Nonnos knüpft an all diese Motive an und adaptiert den Mythos für sein DionysosEpos, indem er eigene Schwerpunkte setzt und Altbekanntes in neuem, dionysischem Licht erscheinen lässt. Die ausführlichste Schilderung des Ganymed-Mythos erfolgt an prominenter Stelle anlässlich der Ekphrasis von Dionysos' Schild im 25. Buch (Nonn. D. 25,429-450), ${ }^{60} \mathrm{im}$ Zuge derer die Entführung des Ganymed durch Zeus sowie seine

57 Vgl. Chrétien (1985) 147 Anm. ad 250-263.

$58 \mathrm{Zu}$ den literarischen und bildlichen Quellen des Ganymed-Mythos vgl. RE VII/1, 737 - 749; Roscher Bd. I/2, 1595-1603; Davidson (2007) 169-200.

59 Vgl. Chrétien (1985) 151f. Anm. ad 309-320 mit Stellenverweisen; Gigli Piccardi (2003) 709; Hernández de la Fuente (2008) 81, 85.

60 Zu Ganymed in den Dionysiaka vgl. Stegemann (1930) 169 f.; Chrétien (1985) 147 f. Anm. ad 259; Gigli Piccardi (2003) 710 Anm. ad 256-259; Agosti (2004) 122f. Anm. ad 429-450, 233 Anm. ad 241 -251, 480 f. Anm. ad 64-104; Spanoudakis (2014a) 363-367. 
Bestellung zum Mundschenk dargestellt wird. Insbesondere auf der Grundlage dieser Stelle wird die narrative Funktion des Ganymed-Mythos in den Dionysiaka greifbar, welche zur Einbettung des Mythos in den dionysischen Kontext dient. Der Szene geht die Beschreibung Thebens unmittelbar voraus (Nonn. D. 25,413-428), auch Maionien, das Geburtsland des Weingottes, wird genannt (Nonn. D. 25,451). Ein charakteristischer Zug der Aneignung tradierter Mythen durch Nonnos ist die Einordnung in und die Anpassung an die Hauptlinie der Erzählung, das Schicksal des Dionysos und des Dionysischen schlechthin. Ganymed, dem die Versorgung der Olympier mit dem Unsterblichkeit garantierenden Nektartrank obliegt, ist das Gegenstück zu Dionysos und dem Wein. Mehrmals in den Dionysiaka erscheint Ganymed als Mundschenk im Olymp, stets wird der Nektar dem Wein als Äquivalent gegenübergestellt (Nonn. $D$. 14,430 - 437; 17,74-80; 27,244-249; 39,62-73; 47,52f., 97f.). Ein weiteres Motiv, das sowohl in den Dionysiaka als auch vor Nonnos greifbar wird, ist die Eifersucht Heras gegenüber dem Zeus-Liebling und Emporkömmling Ganymed (vgl. etwa Verg. Aen. 1,28; Nonn. D. 8,91-102; 31,252-258). ${ }^{61}$ Eine ausgeprägte Beziehung zur dionysischen Sphäre liegt schließlich in der pantomimischen Darbietung Marons, der in einem Ausdruckstanz die mythischen Figuren Ganymed und Hebe darstellt (Nonn. $D$. 19,214-218), wie auch im Wettstreit zwischen den Göttern Eros und Hymenaios, den Ganymed als Schiedsrichter zugunsten des Eros entscheidet (Nonn. D. 33,64-104). ${ }^{62}$

In der Ampelos-Episode wird Ganymed insgesamt fünfmal zum Vergleich mit dem Satyrn herangezogen (Nonn. D. 10,256-259, 309-320; 11,134-139, 289-295; 12,103113), wobei vor allem drei Motive hervortreten: (1.) Ganymed als Götterliebling, (2.) als Repräsentant eines göttlichen Getränks sowie (3.) sein Aufstieg in den Olymp. Zunächst wird Ganymed in der Bittrede des Dionysos als Favorit des Göttervaters sichtbar (Nonn. D. 10,256-259): ${ }^{63}$

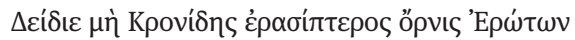

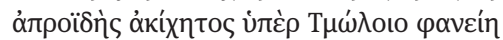

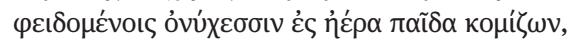

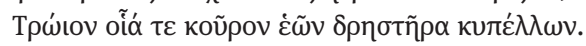

Er [sc. Dionysos] fürchtete, dass der Kronide als Vogel mit den Liebesflügeln der Eroten unvorhergesehen und unerreichbar über dem Tmolos erscheinen und schonungsvoll mit seinen Klauen den Knaben in die Luft heben könnte, genauso wie den Sohn des Tros, den Bereiter seiner Becher.
\end{abstract}

Nonnos setzt Ganymed hier in die Nähe zweier weiterer Vergleichsfiguren für seinen Satyrn: Hyakinthos (Nonn. D. 10,250 - 255) und Pelops (260-263), denen allen durch

61 Im Falle von Nonn. D. 8,91 - 102 ist der Zorn Heras gegen den kleinen Dionysos gerichtet; in Vers 94 wird explizit Ganymed als Parallelfall für einen, wie die Göttermutter meint, unrechtmäßig in den Olymp Aufgestiegenen angeführt; zum Topos von Heras Eifersucht auf Ganymed vgl. auch AP 9,77. 62 Zur literarischen Tradition, der Nonnos in der Szene mit Eros, Hymenaios und Ganymed folgt - er nimmt sich vor allem Apollonios Rhodios zum Vorbild -, siehe Gerlaud (2005) 37-47.

63 Zur Stelle vgl. Hollis (1994) 53. 
die Liebe eines Gottes eine Vergöttlichung zuteil wurde. Während Ganymed hier nur als „Sohn des Tros“ bezeichnet wird, begegnet der Name Ganymed erstmals am Ende einer weiteren Dionysos-Rede (Nonn. D. 10,309-320):

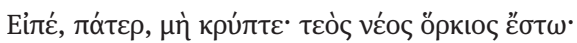

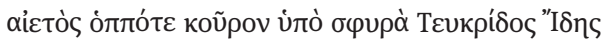

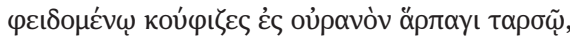

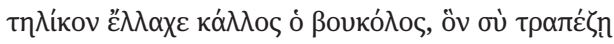

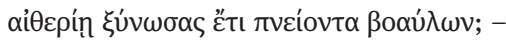

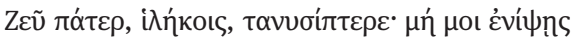

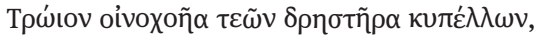

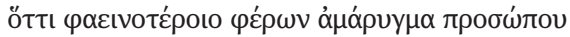

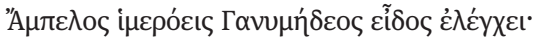

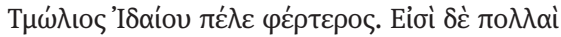

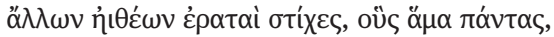

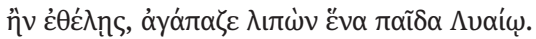

Sprich, Vater! Verhehle nichts! Dein Junge sei die Bestätigung des Schwures! (310) Als du als Adler den Knaben am Fuß des teukrischen Ida schonungsvoll mit räuberischer Klaue in den Himmel emporhobst, war da der Rinderhirt von ebensolcher Schönheit, den du der himmlischen Tafel zuführtest, obwohl er noch die Luft aus dem Rinderstall atmete? Zeus, Vater, sei gnädig, Langflügeliger! Tadle mir nicht (315) den troischen Weinschenk, den Bereiter deiner Becher, weil Ampelos ein funkelnd strahlendes Antlitz besitzt, der Liebliche, und das Aussehen des Ganymed übertrifft! Der vom Tmolos ist besser als der vom Ida! Und es gibt viele reizvolle Scharen von anderen Jünglingen. Die liebe alle zusammen, (320) wenn du willst, aber lass dem Lyaios einen einzigen Knaben!

Mit dem Vergleich zwischen Ganymed und Ampelos passt Nonnos den bekannten Mythos in den neuen, dionysischen Kontext ein. Durch die beiden Vergleichsmomente, Ampelos' außerordentliche Schönheit und den Raub des Knaben durch Zeus, setzt Nonnos den Dionysos-Liebling positiv gegen Ganymed ab und billigt ihm göttlichen Status zu. Nachdem der Dichter zu Beginn der Dionysos-Rede für Ampelos noch einen regelrechten Katalog von Vergleichsfiguren aufgeboten hat, lässt er am Ende lediglich Ěv $\alpha \alpha \tilde{\delta} \delta \alpha$, nämlich Ampelos selbst, übrig (Nonn. D. 10,320). Nonnos, der vor dem Hintergrund von Ganymeds Schicksal seine eigene Geschichte um einen mythischen

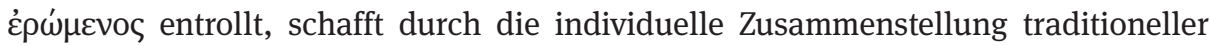
Motive ein neues Narrativ, welches er im 11. Buch fortsetzt und mit effektvoller Rhetorik verbindet: Ate, die personifizierte Verblendung, treibt in ihrer Rede den Satyrn so weit, dass er sich leichtsinnig dem Spiel mit dem Stier hingibt (Nonn. D. 11,134-139):

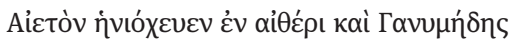

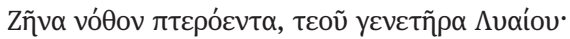

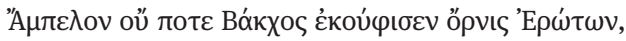

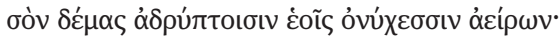

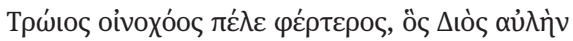

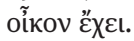


Als einen Adler lenkte am Himmel auch Ganymed (135) Zeus in falscher, geflügelter Gestalt, den Vater deines Lyaios. Bakchos hat Ampelos niemals als Vogel der Eroten emporgehoben, indem er deinen Körper mit seinen nicht zerkratzenden Klauen hochhob. Der troische Weinschenk war stärker, der nun den Hof des Zeus zum Haus hat.

Der Psogos bietet als Hauptvergleichspunkt zwischen Ganymed und Ampelos den Akt der Entführung und den damit einhergehenden Aufstieg eines Sterblichen in den Götterhimmel. In seiner ersten Klagerede auf den zu Tode gekommenen Ampelos lässt Nonnos Dionysos zweimal Ganymed als Vergleichsfigur heranziehen, beide Male als positives Vorbild für den Satyrn und dessen Aufnahme in den Olymp (Nonn. D.11,293295). ${ }^{64}$ Neben der Liebes- und Entführungsthematik ist insbesondere ein Vergleichspunkt essentiell zum Verständnis der Ampelos-Figur: die Verbindung Ganymeds mit dem göttlichen Getränk des Nektars. Ganymed wird in seiner Funktion als göttlicher Mundschenk auf der vierten prophetischen Tafel im Palast des Helios beschrieben und folgt somit unmittelbar auf die Darstellung des Ampelos und des Weines auf der dritten Tafel (Nonn. D. 12,103-117). ${ }^{65}$ Die Kombination der Darstellung des Ganymed und der prophetischen Beischrift, die Dionysos' wichtigstes Attribut, den Wein, ankündigt, ermöglicht es, den Zeus-Liebling dem Ampelos gegenüberzustellen. So wie Ganymed als Liebling des Göttervaters gilt, ist Ampelos Dionysos' favorisierter Begleiter, und genauso wie Ganymed den mit Nektar gefüllten Becher dem obersten Gott darreicht, ${ }^{66}$ wird der Satyr Dionysos mit seinem wichtigsten Attribut ausstatten. Das aus dem traditionellen Mythos bekannte Paar Zeus und Ganymed dient als Folie für Dionysos, und die sonst unbekannte Ampelos-Figur gewinnt durch den Vergleich mit Ganymed an Profil. Die außerordentliche Bedeutsamkeit dieser Figurenverbindung für das narrative Ziel der Ampelos-Episode ist daran abzulesen, dass die Inhalte der beiden Tafeln schon zuvor in Nonn. D. 12,37-40 angekündigt werden und auch hier Ganymed zum Vergleich herangezogen wird.

Ein Anknüpfungspunkt an literarische Traditionen, die Nonnos in der Rekapitulation des Ampelos-Mythos anklingen lässt, liegt in der äußeren Schönheit und erotischen Ausstrahlung des Satyrn, ein poetisches Muster, das für Ganymed spätestens ab dem Hellenismus greifbar wird: ${ }^{67}$ In Call. Epigr. 52 Pfeiffer (= 6 Asper = AP 12,230) wird in einem Atemzug mit dem Götterliebling Ganymed ein sterblicher Knabe genannt, zu dem sich das lyrische Ich hingezogen fühlt. Außerdem finden sich etliche Beispiele von Synkriseis von jungen Männern mit Ganymed in der Anthologia Graeca,

64 Für Text und Übersetzung der Stelle siehe S. 127 f. - Vgl. Stegemann (1930) 169: „Ampelos ist also als irdisches Abbild des Ganymed aufzufassen, wobei der Nachdruck auf den Gedanken des Spendens zu legen ist, was Ampelos mit seiner Metamorphose in die Traube tut.“

65 Für Text und Übersetzung der Stelle siehe S. 194f. - Die Entsprechung von Wein und göttlichem Nektar ist ein häufig angewandtes Motiv in den Dionysiaka; siehe Nonn. D. 7,76 - 78, 134f. und 337 f.; 12,158f.; 17,74-77; 39,64-66; 47,76-78 und $97 \mathrm{f}$. sowie 48,976f.

66 Vgl. Nonn. D. 25,429-450, bes. 445.

67 Zur Beliebtheit der Ganymed-Figur in der Epigrammatik vgl. Agosti (2004) 122 f. Anm. ad 429450. 
wobei teils die erotische Konnotation (AP 12,37,133, 254), teils das Motiv der Angst vor der Entführung durch Zeus vorherrschend ist (AP 65,69). ${ }^{68}$ In AP 12,37,133 und 254 wird zudem der junge Mann seinem mythischen Pendant Ganymed vorgezogen, genauso verfährt auch Nonnos im Vergleich Ganymeds mit Ampelos. Nonnos bedient sich auch mit der katalogartigen Aufzählung von pueri dilecti einer geläufigen literarischen Schreibart. So werden etwa in Pindars 1. Olympischer Ode Ganymed und Pelops einander gegenübergestellt (Pi. 0. 1,36-45), Theokrit seinerseits gesellt den beiden Adonis hinzu (Theoc. 15,124-142). ${ }^{69}$ Von besonderem Interesse für die Konzeption der

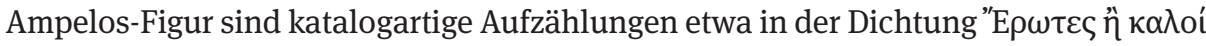
des Phanokles oder auch eine in einem Papyrus-Fragment erhaltene elegische Dichtung (POxy. 3723 = SSH 1186), die einen Katalog von Liebschaften von Göttern mit sterblichen jungen Knaben enthält. ${ }^{70}$ Im Zentrum stehen homoerotische Liebesbeziehungen, die jeweils den Ausgangspunkt für ein Aition bilden ${ }^{71}$ und neben dem Sänger Orpheus und seinem geliebten Kalais auch Tantalos und Ganymed, Agamemnon und Argynnos aufbieten. Mit der Nennung von Apoll und Hyakinthos, Herakles und Hylas sowie möglicherweise wiederum Agamemnon und Argynnos liefert der Papyrus Ähnliches. Ein weiteres Paar bilden Dionysos und ein nicht namentlich

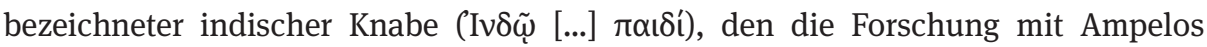
identifiziert, ${ }^{72}$ eine Gleichung, die jedoch nicht unbedingt aufgeht, da bei Nonnos Ampelos an keiner Stelle mit Indien in Verbindung gebracht wird. Bei der Heranziehung derartiger Passagen als tatsächliche Quellentexte für die Dionysiaka ist Vorsicht geboten, bezieht das Epos seine poetische Ausdruckskraft doch aus dem Dialog mit ganzen literarischen Gattungen und Schreibweisen und nicht nur aus einem exakt zu bestimmenden Autor oder Text und erhebt somit den Anspruch, einer universalen dionysischen Poesie Genüge zu leisten. ${ }^{73}$

Als weitere Aspekte des Ganymed-Mythos werden in der Ampelos-Figur der Aufstieg in den Himmel und die Aufnahme unter die Götter reflektiert. ${ }^{74}$ Schon in Ovids

68 Eine Anspielung auf den Ganymed-Mythos findet sich zudem in AP 5,65, das Motiv der Entführung auch in AP 11,330, 407 und 12,220f. Ganymed tritt auch an zwei Stellen der Posthomerika des Quintus Smyrnaeus in Erscheinung: In Q. S. 8,427-442 blickt Ganymed vom olympischen Götterhimmel auf das untergehende Troia und stimmt eine Klage an, und in Q. S. 14,325 f. markiert ein Heiligtum, das die Troianer zu Ehren des Ganymed errichtet haben, die Stadt Troia.

69 Vgl. Chrétien (1985) 147 Anm. ad 250-263.

70 Vgl. Parsons (1988); Gärtner Th. (2008) mit weiteren Literaturangaben.

71 Siehe z. B. einige Idyllen Theokrits, vgl. Hunter (1996) 167-195.

72 Vgl. Parsons (1988) 70 f.; Gärtner Th. (2008) 21.

73 Ganymed ist zusammen mit anderen Knaben und Götterlieblingen ein beliebtes Thema in der spätantiken Kunst und oft Teil des reichen mythologischen Figurenprogramms von Mosaikzyklen, vgl. Blázquez (1993) 52, 398, er tritt außerdem in der römischen Sarkophagkunst in Erscheinung, vgl. Koch u. Sichtermann (1982) $146 \mathrm{f}$.

74 Vgl. Byre (1976) 194f., 286. - Die Vorstellung der Erhebung der Seele in den Himmel ist auch aus dem Platonismus und Neuplatonismus bekannt und schwingt vielleicht auch in der Ampelos-Figur in den Dionysiaka mit: In Pl. Phdr. 255b-c wird, um die physischen Vorgänge bei Verliebtheit zu illus-

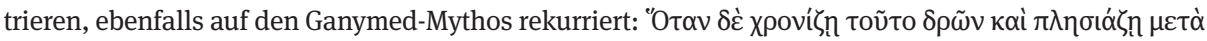


Fasten wird Ampelos als Sternbild des Vindemitor an den Himmel versetzt (Ov. fast. 3,403-414). ${ }^{75}$ Ein Grund für die Wahl Ganymeds als Pendant zu Ampelos mag auch aus Überlieferungen wie den Odyssee-Scholien erklärlich sein, denen zufolge der Ganymed-Mythos mit dem Weinstock in Verbindung steht: Zeus habe Tros, dem Vater des Ganymed, zum Trost keine Pferde, sondern einen goldenen Weinstock überlassen, der an Priamos und schließlich an dessen Neffen Eurypylos weitergegeben wird (Hom. Schol. Od. 11,521).

Nonnos bietet in den Dionysiaka sämtliche Spielarten des Ganymed-Mythos, das Spektrum reicht von elliptischen Andeutungen in der Ampelos-Episode über die volle Ausformulierung in der Schildbeschreibung bis hin zur Umarbeitung in der Szene der pantomimischen Darstellung. Ampelos ist gleichsam als neuer Ganymed Repräsentant einer dionysischen Dichtung, die ihre Vorbilder stets nur zum Ausgangspunkt für eine neue Art des Dichtens nimmt. Nonnianisches Dichten versteht sich als Auseinandersetzung mit den Ausdrucksmöglichkeiten der griechischen Dichtung seit Homer, wobei dem Rezipienten ein größtmögliches Spektrum an Zugangsweisen, gewissermaßen ein erschöpfender Fundus antiken Dichtens und Denkens geboten wird, um altbewährten und ererbten Erzählmustern neue poetische Stilprinzipien entgegenzuhalten.

\subsection{Hylas}

Eine bewusste und kritisch-differenzierte Auseinandersetzung mit Vorbildern in der griechischen Dichtung liegt auch in der Wiederaufnahme hellenistischer Themen vor. Als weitere mythische Vergleichsfigur für Ampelos wird Hylas, der Geliebte des Herakles, ein einziges Mal in den Dionysiaka, und zwar in der Ampelos-Episode, namentlich genannt: ${ }^{76}$ Nach dem Tod des jungen Satyrn eilt Dionysos zum Leichnam und wird bei dieser Gelegenheit Herakles gegenübergestellt, der auf der Suche nach Hylas ist (Nonn. D. 11,226-230). ${ }^{77}$ Die Angleichung von Hylas und Ampelos schafft der

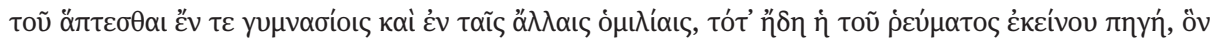

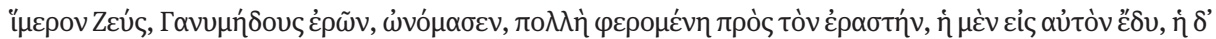

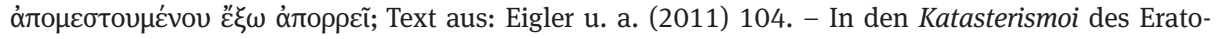
sthenes wird Ganymed mit dem Sternbild des Hydrochoos, des Wassermanns, identifiziert (Eratosth. Cat. 26); vgl. Roscher Bd. I/2, $1596 \mathrm{f}$.

75 Zum Mythos bei Ovid siehe Kap. 3.

$76 \mathrm{Zu}$ den Quellen des Hylas-Mythos siehe RE IX/1, 110-115.

77 Für Text und Übersetzung der Stelle siehe S. 123 f.; zur Interpretation siehe auch Fayant (2012). Auch Ampelos wird an einer Stelle mit Herakles geglichen: Im Rahmen des Ringens mit Dionysos knüpft Nonnos in Nonn. D. 10,373-378 an die Traditionen hellenistischer Dichtung an und verweist auf einen Ringkampf zwischen Zeus und Herakles, der ansonsten nur bei Lykophron belegt ist (Lyc. Alex. 40 - 42). Die Tatsache, dass bei Nonnos der Gott zugunsten seines Lieblings von seinem Sieg zurücktritt, dient der Bestätigung und Etablierung seiner eigenen göttlichen Fähigkeiten. Für die Dionysos-Figur der Dionysiaka bedeutet dieser Vergleich einen weiteren Schritt in Richtung Aner- 
Dichter zunächst durch intratextuelle Bezüge, indem er das Motiv des Neides aus Nonn. D. 11,228 im Laufe der Episode mehrmals anwendet, so im 10. Buch mit den

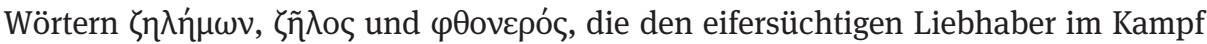
um die Gunst des Ampelos bezeichnen. ${ }^{78}$ In Buch 11 wird das Neid-Motiv mit den Todesschicksalen von Ampelos und Karpos verbunden, ${ }^{79}$ und in Buch 12 tritt Apoll „neidvoll“ gegen Dionysos auf, welcher seit der Metamorphose des Ampelos über das vielseitig einsetzbare Attribut des Weinstocks verfügt. ${ }^{80}$ Indem beide das charakteri-

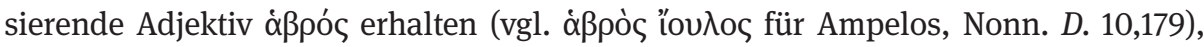
rücken Hylas und Ampelos auch im äußeren Erscheinungsbild eng zusammen. ${ }^{81}$

Durch die Wiederaufnahme von Themen, die aus der hellenistischen Auseinandersetzung mit dem Hylas-Mythos geläufig sind, verfeinert Nonnos Ampelos' Charakterbild. Das Motiv der Schönheit des jugendlichen Geliebten ist in den Hylas-Episoden der hellenistischen Dichter, namentlich bei Apollonios Rhodios (A. R. 1,12071272) und Theokrit (Theoc. 13), ebenso zu finden wie dessen unerwarteter Tod, die Suche durch den Liebhaber und die Trauer um den unwiederbringlichen Verlust. ${ }^{82}$ Von besonderer Bedeutung ist das Leitmotiv des Hylas-Mythos, das Element Wasser, das als Kompositionselement dient: ${ }^{83}$ beim Spiel der Satyrn (Nonn. D. 10,139-174), in den Schwimm-Agonen von Dionysos und Ampelos (11,43-55) sowie von Kalamos und Karpos (400 - 426). Um Anklänge an die Hylas-Szene bei Apollonios handelt es sich auch beim Botenbericht eines anonymen Satyrn an Dionysos (Nonn. D. 11,224f.): ${ }^{84}$ Das Schicksal des Jungen - die Liebesbeziehung zu einem (Halb-)Gott, der Tod und die Apotheose - entspricht dem Schicksal des Ampelos, die Reaktion des Herakles der des Dionysos: Beide begeben sich gezeichnet von der Unglücksmeldung auf die Suche nach dem Vermissten.

kennung als Gott; vgl. Del Corno u. a. (1997) 308; Chrétien (1985) 155 Anm. ad 377; Gigli Piccardi (2003) 724 Anm. ad 373-377.

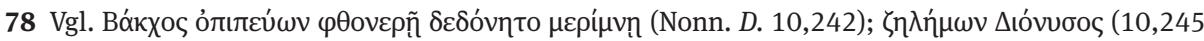

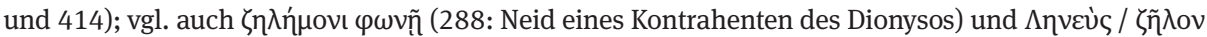

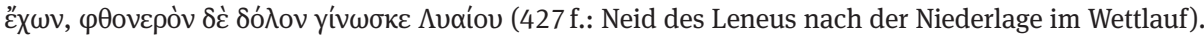

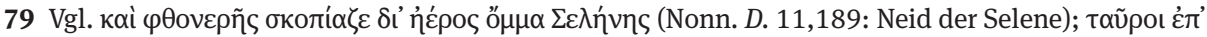

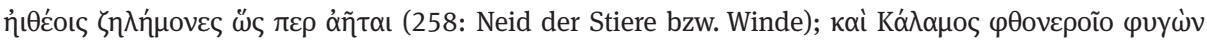

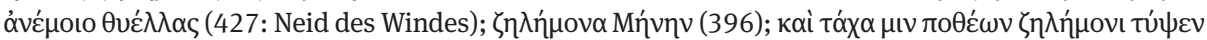

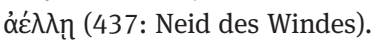

80 Vgl. Фoíß

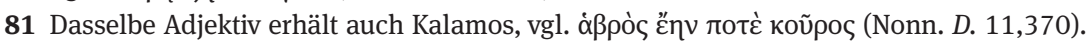

82 Zur Schönheit des Hylas siehe A. R. 1,1229-1232; Theoc. 13,7. Zum Trauer-Motiv, das Theokrit auf Hylas überträgt, vgl. Theoc. 13,53 f. - Zur Hylas-Episode bei Apollonios Rhodios und Theokrit vgl. Köhnken (1965) 9-83; Fuchs (1969); Mauerhofer (2004) 37 - 112. - Zum Hylas-Mythos in der Ampelos-Episode siehe Mazza (2012) 105-117, der betont, dass es Nonnos nicht nur um motivische Übernahmen, sondern vor allem um literarische Anspielungen auf konkrete Stellen hellenistischer Dichter geht.

83 Zum Element Wasser in der Ampelos-Episode siehe S. 25-28 sowie Kröll (2013); zum Motiv Wasser und zu dessen narrativer Funktion in den Idyllen Theokrits vgl. Segal (1981).

84 In A. R. 1,1240-1260 vernimmt Polyphem den Hilfeschrei des Hylas und warnt Herakles. 
Zusätzlich zu diesen allgemeinen, aber für Hylas typischen Themen bezieht sich Nonnos auch auf konkrete Referenztexte, schafft so ein Netz von intertextuellen Bezügen und koppelt seine Ampelos-Episode an die literarische Tradition. Das Motiv der

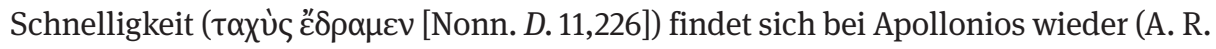

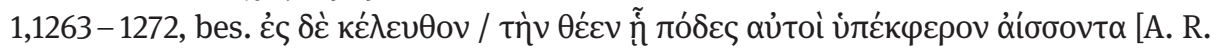
1,1263f.]). ${ }^{85}$ Besonderen Nachdruck verleiht Apollonios der Situation durch ein Gleichnis: Herakles stürzt wie ein von einer Bremse gestochener Stier in Richtung Hylas (A. R. 1,1265-1272), dies erinnert an den Stier in Buch 11 der Dionysiaka, der Ampelos zum Verhängnis wird (Nonn. D. 11,188-223). ${ }^{86}$ Ähnlich wie die Figuren wird von Nonnos auch die Örtlichkeit auf seine Geschichte übertragen: Auf der Suche nach dem verschollenen Ampelos irrt Dionysos durch gebirgiges Terrain genauso wie He-

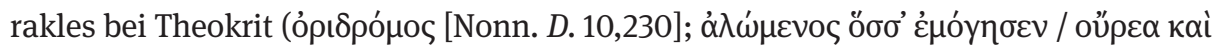

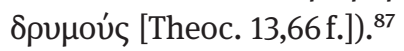

In der Szene mit Ampelos’ Tod schöpft Nonnos, wie Francis Vian beobachtet, zu einem Großteil aus der Europa des Moschos. ${ }^{88}$ Diese Anlehnung an das hellenistische Vorbild wird auch in der vorliegenden Stelle sichtbar: Das „räuberische Mädchen“ (а̋ [190]), der für Ampelos' Tod verantwortlich ist, und rekapituliert zugleich die Ent-

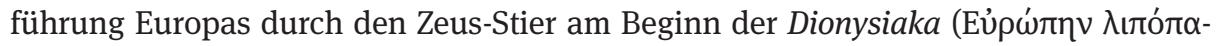

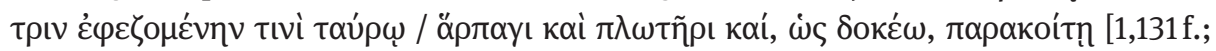
vgl. auch Nonn. D. 7,118]).

Auf den Hylas-Mythos wird ein weiteres Mal in der Geschichte um Ampelos angespielt, und zwar in der katalogartigen Aufzählung der Unternehmungen, die der Satyr oftmals gemeinsam mit Dionysos startet. Im Zentrum steht das Wasser-Motiv, das dem Dichter als Vehikel für eine abermalige Anknüpfung an die hellenistische Dichtung dient (Nonn. D. 10,226-229): ${ }^{89}$

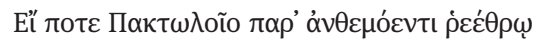

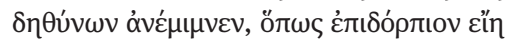

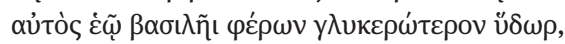

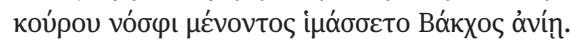

Wenn er [sc. Ampelos] einmal am blumengeschmückten Strom des Paktolos säumend verweilte, um nach dem Essen selbst seinem König süßeres Wasser zu bringen, wenn der Knabe fern war, dann verzehrte sich Bakchos vor Trauer.

85 Zum Motiv Schnelligkeit vgl. Fayant (2012) 4 und 6.

86 Theokrit bietet im Gegensatz dazu ein Löwengleichnis (Theoc. 13,61-64).

87 Vgl. Vian (1995) 168; Gigli Piccardi (2003) 774f. - Auch Apollonios deutet als Ort des Geschehens

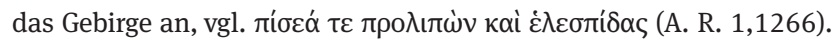

88 Vgl. Vian (1995) 8 und Anm. ad 1,162 f.; Del Corno u. a. (1997) 314; Gigli Piccardi (2003) 765; Fayant (2012) $18 \mathrm{f}$.

89 Vgl. Del Corno u. a. (1997) 305 und 315. 
Obwohl weder Hylas noch Herakles mit Namen genannt werden, können diese Figuren mühelos assoziiert werden. Mit dem Motiv des Wasserholens sind wörtliche Anklänge an Apollonios und Theokrit festzustellen (A. R. 1,1207-1210; Theoc. 13,36-39): Das

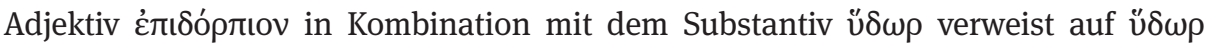

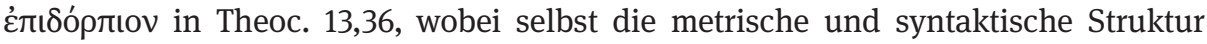

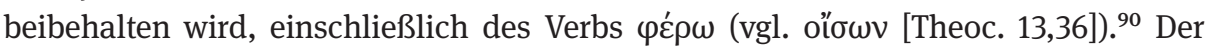
Hylas-Mythos bei Theokrit ist auch im Hinblick auf die endgültige Bestimmung der Jünglinge aufschlussreich: Am Schluss des genannten Idylls erfolgt die Aufnahme des

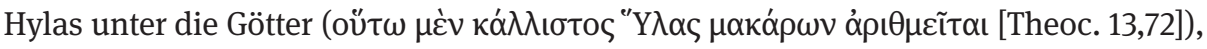
ganz ähnlich wie auch Ampelos durch die Metamorphose in den Weinstock zu einem Wesensteil des Gottes Dionysos wird. Auch das Motiv der Apotheose ist somit in der Geschichte um Herakles und Hylas vorgezeichnet. ${ }^{91}$

\subsection{Europa, Atymnios, Glaukos und Bellerophon}

Die Reprise des hellenistischen Wort- und Themenbestandes bewerkstelligt Nonnos zugleich auf thematischer, syntaktischer, metrischer und lexikalischer Ebene, wobei er nicht bloß imitiert, sondern durch den innovativ-kreativen Ansatz in produktive Konkurrenz zu seinen Vorbildern tritt. Die Stiere, auf deren Rücken Ampelos und Europa sitzen, sind ihrem Wesenskern nach die Götter Zeus und Dionysos selbst (Nonn. D. 11,152-154):

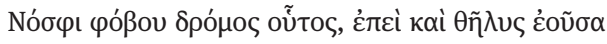

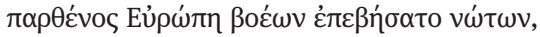

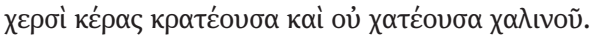

Fern jeglicher Furcht soll dir dieser Ritt sein, da ja auch, obwohl sie eine Frau war, das Mädchen Europa auf den Rücken des Stieres stieg, mit den Händen das Horn festhielt und keine Zügel benötigte.

Mit der Parallelisierung der beiden Reiter geht auch diejenige der beiden Stiere einher; genauso wie es sich beim Stier Europas eigentlich um Zeus handelt, steckt in Ampelos' Stier Dionysos. ${ }^{92}$ Die Gegenüberstellung von Europa und Ampelos fügt sich in die

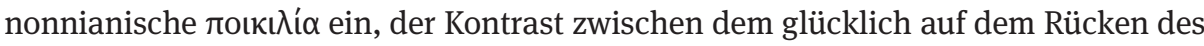
Zeus-Stieres sitzenden Mädchen und dem zu Tode getriebenen Ampelos könnte nicht größer sein.

90 Vgl. Fayant (2012) 8-11.

91 Eine weitere Stelle der Ampelos-Episode erinnert an die Hylas-Episode: In der Kalamos-KarposErzählung wird durch das Motiv des Hilferufes ebenfalls auf den Raub des Hylas durch Nymphen angespielt (Nonn. D. 11,390, 449, 454; Theoc. 13,58-60), vgl. Fayant (2012) 24.

92 Zum Stier als dem Tier des Dionysos vgl. Daniélou (1992) 112-116; Otto (2011) 150-152. 
Ritt und Wagenfahrt bilden zudem die Hauptvergleichspunkte zwischen Ampelos und drei weiteren Figuren. In Dionysos' erster Klagerede wird als mythische Parallele für Ampelos neben Hyakinthos auch Atymnios, ein Liebling Apolls, präsentiert, der durch eine missglückte Wagenfahrt zu Tode kommt (Nonn. D. 11,255-263). Dionysos preist Apoll glücklich, da dieser nach dem Tod seines Geliebten Atymnios wenigstens dessen Namen übernehmen konnte und nun das Epitheton Atymnios trage (Nonn. D. 11,258f.). ${ }^{93}$ Nonnos verschränkt die Schicksale beider Apoll-Geliebten miteinander, doch während er den Hyakinthos-Mythos ausführlich wiedergibt, deutet er die Geschichte um Atymnios lediglich durch das Epitheton an. Wiederum werden mythische Traditionen mit Episoden in den Dionysiaka gekoppelt. Die Tatsache, dass Apoll nach dem Tod seines geliebten Jünglings dessen Namen als Epitheton erhält, ist außer bei Nonnos nirgendwo belegt. ${ }^{94}$ Nonnos greift einen Mythos auf, der aufgrund der knappen Andeutungen als beim Publikum bekannt gelten darf, und überträgt diesen auf seine eigene Geschichte. Zentrale poetische Verfahrensweisen wie die Übertragung des Namens des Geliebten auf die Gottheit übernimmt er, indem er ein neues Epitheton für Apoll konstruiert, und zwar nach dem Vorbild des Dionysos. Nonnos erscheint als autonome künstlerische Instanz, wenn er, um seinen Dionysos als Gott zu legitimieren, nach dessen Vorbild einen gleichfalls neuen Beinamen für einen bereits etablierten Gott kreiert. Die Legitimierung des neuen Helden durch den Mythos entsteht in diesem Fall nicht durch Hinzuziehen alter Traditionen aus der klassischen oder hellenistischen Dichtung, sondern aus dem augenblicklichen künstlerischen Bedürfnis heraus.

Und noch einmal wird Atymnios zum Spiegelbild für Ampelos: Neben Ganymed bringt Ate in ihrer Rede an Ampelos auch Apoll und seinen Geliebten zur Sprache (Nonn. D. 11,130 f.): ${ }^{95}$

93 Zur Figur Atymnios allgemein vgl. Apollod. 3,1,2; RE II/1, 2261 (Nr. 4); zu Atymnios bei Nonnos siehe Chuvin (1991) 57 f.; Gigli Piccardi (2003) 762; Agosti (2004) 290; Atymnios findet sich in Gesellschaft von weiteren Geliebten des Apoll gemeinsam mit Ampelos und Dionysos in der Liste der männlichen Göttergeliebten in den Pseudo-Klementinen, siehe S. $42 \mathrm{f}$.

94 Vgl. Del Corno u. a. (1997) 316.

95 Atymnios erscheint ein weiteres Mal in den Dionysiaka: In Nonn. D. 19,180-188 gibt Maron anlässlich der Leichenspiele des Staphylos eine Tanzdarbietung zum Besten, stellt sich in einer Rede selbst vor und legt seine Position dar: Er bezeichnet sich als „Diener des Bakchos“ und setzt sich

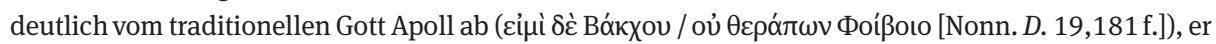
wolle keine Klagelieder anstimmen, so wie Apoll dies aufgrund des Todes seines geliebten Atymnios

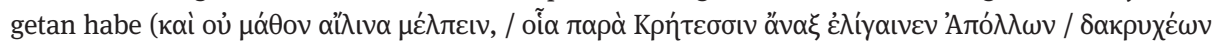

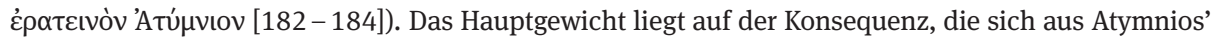
Hybris ergibt, nämlich Sturz und Tod. Untermalt wird diese Stimmung durch die unmittelbar nachfolgende Anspielung auf die Trauer der Heliaden über Phaethon (Nonn. D. 19,184-186), vgl. Gonnelli (2003) 386f. Die pantomimische Darbietung des Maron beschränkt sich aber nicht auf die Trauer um den verstorbenen Staphylos, der Tänzer verkündet, er werde nicht wie Apoll über den Verlust eines geliebten Menschen trauern, sondern einen freudigen Tanz aufführen, „so als ob Staphylos noch am

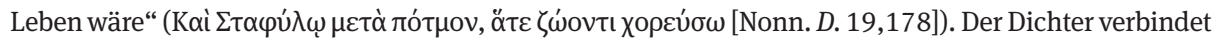
die Szene mit dem eigentlichen Thema seines Epos, Dionysos: Der sorglose und heitere Tanz Marons ist ein bakchischer Tanz, der Tod und Wiederauferstehung des Weingottes spiegelt. Zu Marons Tanz vgl. 


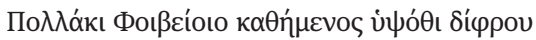

130

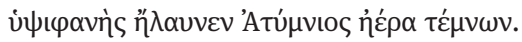

(130) Oftmals saß hoch oben auf dem Wagen des Phoibos Atymnios, zeigte sich in der Höhe und preschte voran, die Luft durchschneidend.

Nonnos fügt Atymnios in ein dichtes Netz an Selbstreferenzen ein: Der in Nonn. $D$. 11,257 genannte Windgott Zephyr, durch den Atymnios zu Tode kommt, ist schon aus dem Hyakinthos-Mythos bekannt, das Motiv des neidischen und Tod bringenden Windstoßes findet sich auch im Zusammenhang mit dem Schicksal des Karpos:

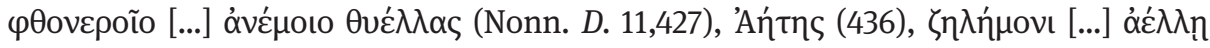
(437). Das unverbrüchliche Band zwischen überkommenen Mythen, unkonventionellen und möglicherweise ad hoc kreierten Varianten sowie gänzlich neuen Umsetzungen ist die Quintessenz nonnianischen Dichtens. Der mythische Typus des Götterlieblings, der durch selbst verschuldete Hybris stirbt, gilt sowohl für Hyakinthos, Atymnios und Ampelos als auch für Phaethon, der an späterer Stelle in den Dionysiaka eine wichtige Rolle spielen wird (Nonn. D. 30,105-434). ${ }^{96}$ Auch der Atymnios-Mythos fügt sich also in die großen Erzähllinien der Ampelos-Episode und der Dionysiaka.

Das Thema des Aufstiegs in den Himmel mithilfe von Wagen oder Pferden wird in der Ate-Rede anhand der Figuren Glaukos und Bellerophon weiter ausgeführt (Nonn. D. 11,143-146). ${ }^{97}$ Der im Mythos des Atymnios bereits angedeutete Gedanke der Hybris und des daraus resultierenden Sturzes wird im Besonderen durch das Schicksal von Glaukos und Bellerophon hervorgekehrt, die beide durch den Ritt auf Pferden sterben: Glaukos, ein Sohn des Sisyphos, wird während der Leichenspiele zu Ehren des Pelias aufgrund seiner Hybris gegen die Göttin Aphrodite von seinen mit Wahnsinn geschlagenen Stuten abgeworfen; ${ }^{98}$ Ähnliches widerfährt Bellerophon, dem Sohn des Poseidon, mit seinem Sturz vom Pegasos. ${ }^{99}$ Der unglückliche Sturz von Pferd oder Wagen wird in den Dionysiaka mehrmals zum Thema gemacht: Bellerophon wird weitere zwei Male als mythisches Exempel für den Sturz einer Figur der Handlung herangezogen (Nonn. D. 28,164-167, im Anschluss daran in Nonn. D. 28,168-171 der

Delavaud-Roux (2009); zu Pantomimus und Gestik in den Dionysiaka siehe auch Weinreich (1948) 163 - 172; Haidacher (1949) 80; Miguélez Cavero (2009a), zum Pantomimus in der Antike allgemein vgl. Hall u. Wyles (2008); Webb (2008) 58 - 94; Weiss (2014) 128 - 135. Pantomimus wird außerdem in Nonn. D. 5,103-107 erwähnt (pantomimischer Tanz Polymnias anlässlich der Hochzeit von Kadmos und Harmonia).

96 Vgl. Gonnelli (2003) $386 \mathrm{f}$.

97 Für Text und Übersetzung der Stelle siehe S. 137.

98 Vian (1995) 162 nennt die Quellen für diesen Mythos: E. Ph. 1123-1127; Str. 9,2,24 (C 408f.); Verg. georg. 3,267 f.; Hyg. fab. 250 und 273,11; Paus. 6,20,19; siehe außerdem den nur fragmentarisch erhaltenen Glaukos Potnieus des Aischylos (A. Fr. 36-42 Radt); vgl. auch Gigli Piccardi (2003) 763 f. 99 Gianfranco Agosti verweist auf die Bedeutung der Figuren Bellerophon und Pegasos im Heidentum der Spätantike als Symbole der menschlichen Seele; die Beliebtheit des Mythos in den nachchristlichen Jahrhunderten lasse sich zudem aus den Zeugnissen der bildenden Kunst und der Literatur erschließen; vgl. Agosti (2004) $263 \mathrm{f} ., 820 \mathrm{f}$. 
Sturz eines kämpfenden Inders; Nonn. D. 38,400-405, nachfolgend der Sturz des Phaethon). In der Ampelos-Episode versinnbildlicht der Sturz des Bellerophon Ampelos' folgenschweres Scheitern durch Hybris und nimmt so das Schicksal des Satyrn vorweg. Der Wahnsinn der tobenden Pferde Glaukons ist analog zum bakchischen Wahnsinn des Stieres zu lesen, der Ampelos zum Verhängnis wird.

\subsection{Marsyas}

Ein mittelbarer Verweis auf Hybris und Fall des Ampelos liegt in Marsyas vor. ${ }^{100}$ Im Rahmen einer längeren Passage über die Wirkung der Liebe auf Dionysos (Nonn. $D$. 10,217-277) erfolgt ein Vergleich des Marsyas mit Ampelos (Nonn. D. 10,230-234):

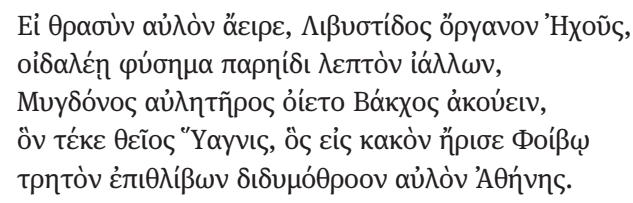

(230) Wenn er den verwegenen Aulos anhob, das Instrument der libyschen Echo, und mit aufgeblasenen Wangen einen sanften Lufthauch hervorbrachte, dann glaubte Bakchos, den mygdonischen Aulosspieler zu hören, den der göttliche Hyagnis gezeugt hatte, der mit Phoibos in einen üblen Wettstreit trat, als er auf die Löcher von Athenes zweistimmigem Aulos drückte.

Der Anblick des Ampelos beim Flötenspiel ruft bei Dionysos die Erinnerung an Marsyas, den „mygdonischen Aulosspieler“, wach, der sich mit seinem Instrument einem musischen Wettkampf gegen Apoll stellte, von diesem jedoch besiegt und zur Strafe gehäutet wurde. Hybris und Todesschicksal bilden die Vergleichspunkte zwischen Marsyas und Ampelos; genauso wie Marsyas an der eigenen Hybris gegen Apoll scheiterte, indem er sich unrechtmäßigerweise die Beherrschung der göttlichen Kunst anmaßte und gegen einen Gott auftrat, geht Ampelos an der vorzeitigen Inanspruchnahme dionysischer Insignien zugrunde.

Durch die Kombination mehrerer Figuren, von denen jede zumindest eine Eigenschaft mit ihm gemeinsam hat, erlangt Ampelos sein volles Charakterbild. Dem Mythos um Marsyas und den im Anschluss geschilderten Schicksalen von Hyakinthos und Ganymed (Nonn. D. 10,250-259) kommt eine wesentliche kompositorische Funktion innerhalb der Ampelos-Episode zu: Sie skizzieren das Schicksal des Ampelos und bereiten so auf die Ankunft des Weines in der Welt vor. Die mythischen Synkriseis für den Satyrn, die über die gesamte Episode verteilt sind, werden zum tonangebenden

100 Marsyas tritt bereits am Ende des Proömions der Dionysiaka unmittelbar vor der Geschichte um Zeus und Europa auf: Nach der Einforderung diverser bakchischer Utensilien wie Thyrsos, Hirschkalbfell und Handtrommeln lehnt der Dichter die traditionellen poetischen Ausdrucksformen - die homerische Epik und die Bukolik mit ihrer Flötenbegleitung - ab, an den in diesem Zusammenhang genannten Aulos wird der Marsyas-Mythos angeschlossen (Nonn. D. 1,41-44). 
Gestaltungselement nonnianischer Poetik. An narrativen Höhepunkten - der Rede der Ate und den beiden Trauerreden des Dionysos im 11., der Ekphrasis der prophetischen Tafeln im Helios-Palast, der Atropos- sowie der Dionysos-Rede im 12. Buch - bedient sich der Dichter mythischer Reminiszenzen für sein Ampelos-Bild. Die beinahe ausschließliche Charakteristik gängiger Mythologeme sowie das weitgehende Fehlen individueller Züge des Ampelos lassen den Schluss zu, dass Nonnos sich hier seinen eigenen, ganz auf die Erfordernisse der Erzählung ausgerichteten Ampelos schafft. Die Funktionalisierung vertrauter Mythen für seinen Ampelos sowie die Transferierung von Bekanntem auf selbst Konzipiertes verleiht dem antiquarischen Wissen neue, schöpferische Impulse: „[...] Nonnos rattache l'épisode d'Ampélos, peu connu par ailleurs, à des antécedents hellénistiques prestigieux que le lecteur érudit ne devait pas manquer de repérer. Il revendique ainsi une filiation littéraire. “ ${ }^{\text {101 }}$ Nonnos verweilt niemals bei einem bloßen Zitieren und Imitieren, mit dem Aufgreifen von Themen und sprachlichen Merkmalen setzt er sich mit seinen literarischen Vorbildern auseinander und bringt gleichzeitig ein neuartiges literarisches Produkt hervor, mit dem er dezidiert in Konkurrenz zur Tradition tritt und sich von dieser abzugrenzen sucht. ${ }^{102}$ 